\title{
Geometric combinatorial algebras: cyclohedron and simplex
}

\author{
S. Forcey • Derriell Springfield
}

Received: 4 September 2009 / Accepted: 31 March 2010 / Published online: 27 April 2010

(C) Springer Science+Business Media, LLC 2010

\begin{abstract}
In this paper we report on results of our investigation into the algebraic structure supported by the combinatorial geometry of the cyclohedron. Our new graded algebra structures lie between two well known Hopf algebras: the MalvenutoReutenauer algebra of permutations and the Loday-Ronco algebra of binary trees. Connecting algebra maps arise from a new generalization of the Tonks projection from the permutohedron to the associahedron, which we discover via the viewpoint of the graph associahedra of Carr and Devadoss. At the same time, that viewpoint allows exciting geometrical insights into the multiplicative structure of the algebras involved. Extending the Tonks projection also reveals a new graded algebra structure on the simplices. Finally this latter is extended to a new graded Hopf algebra with basis all the faces of the simplices.
\end{abstract}

Keywords Hopf algebra · Graph associahedron · Cyclohedron · Graded algebra

\section{Introduction}

1.1 Background: polytope algebras

In 1998 Loday and Ronco found an intriguing Hopf algebra of planar binary trees lying between the Malvenuto-Reutenauer Hopf algebra of permutations [13] and the Solomon descent algebra of Boolean subsets [11]. They also described natural Hopf algebra maps which neatly factor the descent map from permutations to Boolean

S. Forcey $(\bowtie) \cdot$ D. Springfield

Tennessee State University, Nashville, TN 37209, USA

e-mail: sforcey@tnstate.edu

S. Forcey

The University of Akron, 302 Buchtel Common, Akron, OH 44325, USA

e-mail: sforcey@gmail.com 


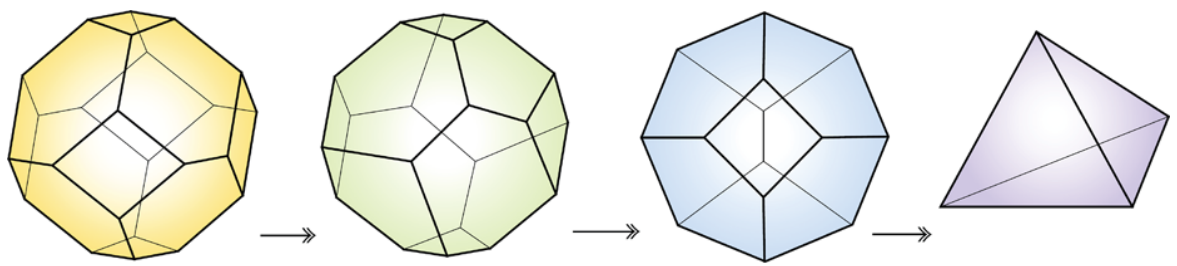

Fig. 1 The main characters, left to right: $\mathcal{P}_{4}, \mathcal{W}_{4}, \mathcal{K}_{4}$, and $\Delta^{3}$

subsets. Their first factor turns out to be the restriction (to vertices) of the Tonks projection from the permutohedron to the associahedron. Chapoton made sense of this latter fact when he found larger Hopf algebras based on the faces of the respective polytopes [5]. Here we study several new algebraic structures based on polytope sequences, including the cyclohedra, $\mathcal{W}_{n}$, and the simplices, $\Delta^{n}$. In Fig. 1 we show the central polytopes, in three dimensions.

We will be referring to the algebras and maps discussed in [2] and [1]. In these two papers, Aguiar and Sottile make powerful use of the weak order on the symmetric groups and the Tamari order on binary trees. By leveraging the Möbius function of these two lattices they provide clear descriptions of the antipodes and of the geometric underpinnings of the Hopf algebras. They also demonstrate cofreeness, characterize primitives of the coalgebras, and, in the case of binary trees, demonstrate equivalence to the non-commutative Connes-Kreimer Hopf algebra from renormalization theory.

Here we generalize the well known algebras based on associahedra and permutohedra to new ones on cyclohedra and simplices. The cyclohedra underlie graded algebras, and the simplices underlie new Hopf algebras. We leave for future investigation many potential algebras and coalgebras based on novel sequences of graph associahedra. The phenomenon of polytopes underlying Hopf structure may be rare, but algebras and coalgebras based on polytope faces are beginning to seem ubiquitous. There does exist a larger family of Hopf algebras to be uncovered in the structure of the polytope sequences derived from trees, including the multiplihedra, their quotients and their covers. These are studied in [9].

\subsection{Background: cyclohedra}

The cyclohedron $\mathcal{W}_{n}$ of dimension $n-1$ for $n$ a positive integer was originally developed by Bott and Taubes [3], and received its name from Stasheff. The name points out the close connection to Stasheff's associahedra, which we denote $\mathcal{K}_{n}$ of dimension $n-1$. The former authors described the facets of $\mathcal{W}_{n}$ as being indexed by subsets of $[n]=1,2, \ldots, n$ of cardinality $\geq 2$ in cyclic order. Thus there are $n(n-1)$ facets. All the faces can be indexed by cyclic bracketings of the string $123 \ldots n$, where the facets have exactly one pair of brackets. The vertices are complete bracketings, enumerated by $\left(\begin{array}{c}2(n-1) \\ n-1\end{array}\right)$.

The space $\mathcal{W}_{n} \times S^{1}$, seen as the compactification of the configuration space of $n$ distinct points in $\mathbb{R}^{3}$ which are constrained to lie upon a given knot, is used to define new invariants which reflect the self-linking of knots [3]. Since their inception the cyclohedra have proven to be useful in many other arenas. They provide an excellent example of a right operad module (over the operad of associahedra) as shown 

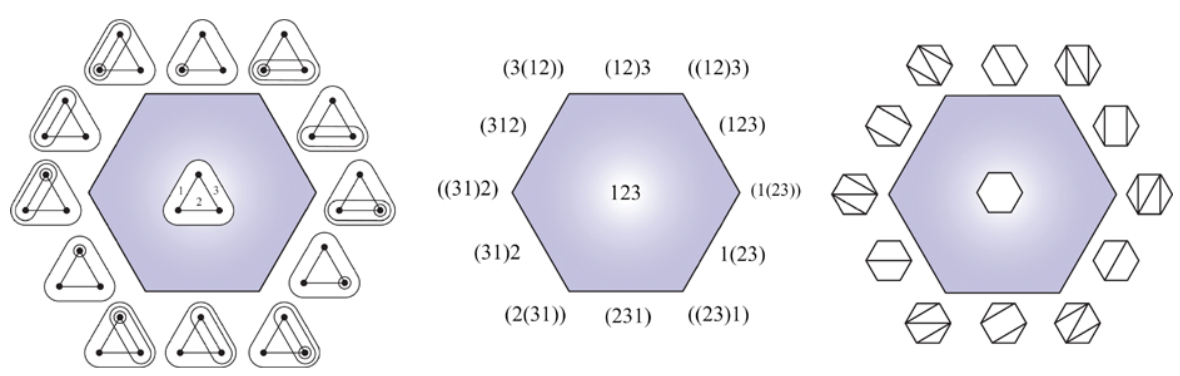

Fig. 2 The cyclohedron $\mathcal{W}_{3}$ with various indexing

in [14]. Devadoss discovered a tiling of the $(n-1)$-torus by $(n-1)$ ! copies of $\mathcal{W}_{n}$ in [7]. Recently the cyclohedra have been used to look for the statistical signature of periodically expressed genes in the study of biological clocks [15].

The faces of the cyclohedra may also be indexed by the centrally symmetric subdivisions of a convex polygon of $2 n$ sides, as discovered by Simion [19]. In this indexing the vertices are centrally symmetric triangulations, which allowed Hohlweg and Lange to develop geometric realizations of the cyclohedra as convex hulls [10]. This picture is related to the work of Fomin, Reading and Zelevinsky, who see the cyclohedra as a generalization of the associahedra corresponding to the $B_{n}$ Coxeter diagrams [18]. From their perspective the face structure of the cyclohedron is determined by the sub-cluster structure of the generators of a finite cluster algebra.

In contrast, for Devadoss the cyclohedra arise from truncating simplex faces corresponding to sub-diagrams of the $\tilde{A}_{n}$ Coxeter diagram, or cycle graph [4]. In this paper we will work from the point of view taken by Devadoss and consider the faces as indexed by tubings of the cycle graph on $n$ vertices. Given a graph $G$, the graph associahedron $\mathcal{K} G$ is a convex polytope generalizing the associahedron, with a face poset based on the full connected subgraphs, or tubes of $G$. For instance, when $G$ is a path, a cycle, or a complete graph, $\mathcal{K} G$ results in the associahedron, cyclohedron, and permutohedron, respectively. In [8], a geometric realization of $\mathcal{K} G$ is given, constructing this polytope from truncations of the simplex. In [4] the motivation for the development of $\mathcal{K} G$ is that it appears in tilings of minimal blow-ups of certain Coxeter complexes, which themselves are natural generalizations of the moduli spaces $\overline{\mathcal{M}}_{0, n}(\mathbb{R})$.

The value of the graph associahedron to algebraists, as we hope to demonstrate here, is twofold. First, a unified description of so many combinatorial polytopes allows useful generalizations of the known algebraic structures on familiar polytope sequences. Second, the recursive structure described by Carr and Devadoss in a general way for graph associahedra turns out to lend new geometrical meaning to the graded algebra structures of both the Malvenuto-Reutenauer and Loday-Ronco algebras. The product of two vertices, from terms $P_{i}$ and $P_{j}$ of a given sequence of polytopes $\left\{P_{n}\right\}_{n=0}^{\infty}$, is described as a sum of vertices of the term $P_{i+j}$ to which the operands are mapped. The summed vertices in the product are the images of classical inclusion maps composed with our new extensions of the Tonks projection. 


\subsection{Summary}

\subsubsection{Notation}

The Hopf algebras of permutations, binary trees, and Boolean subsets are denoted respectively SSym, Y Sym and $\mathcal{Q} S y m$, as in [2] and [1]. Note that some of our sources, including Loday and Ronco's original treatment of binary trees, actually deal with the dual graded algebras. The larger algebras of faces of permutohedra, associahedra and cubes are denoted $\mathfrak{S} \tilde{S} y m, \mathcal{Y} \tilde{S} y m$ and $\mathcal{Q} \tilde{S} y m$. The new algebras of cyclohedra vertices and faces are denoted $\mathcal{W} S y m$ and $\mathcal{W} \tilde{S} y m$. The new algebra of vertices of the simplices is denoted $\Delta S y m$. Finally the new Hopf algebra of faces of the simplices is denoted $\Delta \tilde{S} y m$. Throughout there are three important maps we will use. First we see them as polytope maps: $\hat{\rho}$ is the inclusion of facets defined by Devadoss as a generalization of the recursive definitions of associahedra, simplices and permutohedra; $\eta$ is the projection from the polytope of a disconnected graph to its components; and $\Theta$ is the generalization of Tonks's projection from the permutohedron to the associahedron. This last gives rise to maps $\hat{\Theta}$ which are algebra homomorphisms.

\subsubsection{Main results}

In Theorem 5.3 we demonstrate that $\mathcal{W} S y m$ is an associative graded algebra. In Theorem 6.4 we extend this structure to the full poset of faces to describe the associative graded algebra $\mathcal{W} \tilde{S} y m$. In Theorem 7.3 we demonstrate an associative graded algebra structure on $\Delta S y m$. In Theorem 7.11 we show how to extend this structure to become a new graded Hopf algebra $\Delta \tilde{S} y m$, based upon all the faces of the simplices. Thus its graded dimension is $2^{n}$.

Theorems 4.1, 4.3 and 6.1 and Remark 5.5 point out that the multiplications in all the algebras studied here can be understood in a unified way via the recursive structure of the face posets of associated polytopes. To summarize, the products can be seen as a process of projecting and including of faces, via $\Theta, \hat{\rho}$ and $\eta$. The algebras based on the permutohedron don't use $\Theta$ or $\eta$, and the algebras based on the simplices do not use $\Theta$, but otherwise the definitions will seem quite repetitive. We treat each definition separately to highlight those differences, but often the proofs of an early version will be referenced rather than repeated verbatim. The coproducts of $\mathcal{Y}$ Sym and SSym are also understandable as projections of the polytope geometry, as mentioned in Remarks 4.2 and 4.4.

Before discussing algebraic structures, however, we build a geometric combinatorial foundation. We show precisely how the graph associahedra are all cellular quotients of the permutohedra (Lemma 3.2), and how the associahedron is a quotient of any given connected graph associahedron (Theorem 3.5). Results similar to these latter statements are implied by the work of Postnikov in [17], and were also reportedly known to Tonks in the cases involving the cyclohedron [4].

The various maps arising from factors of the Tonks projection are shown to be algebra homomorphisms in Theorems 5.6, 5.7 and Lemma 7.5. Corollary 5.9 points out the implication of module structures on $\mathcal{W} S y m$ over $\mathfrak{S} S y m$; and on $\mathcal{Y}$ Sym over WSym. 


\subsubsection{Overview of subsequent sections}

Section 2 describes the posets of connected subgraphs which are realized as the graph associahedra polytopes. We also review the cartesian product structure of their facets. Section 3 shows how the Tonks cellular projection from the permutohedron to the associahedron can be factored in multiple ways so that the intermediate polytopes are graph associahedra for connected graphs. We also show that the Tonks projection can be extended to cellular projections to the simplices, again in such a way that the intermediate polytopes are graph associahedra. In particular, we focus on a factorization of the Tonks projection which has the cyclohedron (cycle graph associahedra) as an intermediate quotient polytope between permutohedron and associahedron. In Sect. 4 we use the viewpoint of graph associahedra to redescribe the products in SSym and $\mathcal{Y}$ Sym, and to point out their geometric interpretation. The latter is based upon our new cellular projections as well as classical inclusions of (cartesian products of) lowdimensional associahedra and permutohedra as faces in the higher-dimensional polytopes of their respective sequences. In Sect. 5 we begin our exploration of new graded algebras with the vertices of the cyclohedron. Then we show that the linear projections following from the factored Tonks projection (restricted to vertices) are algebra maps. We extend these findings in Sect. 6 to the full algebras based on all the faces of the polytopes involved. Finally in Sect. 7 we generalize our discoveries to the sequence of edgeless graph associahedra. This allows us to build a graded algebra based upon the vertices of the simplices. By carefully extending that structure to the faces of the simplices we find a new graded Hopf algebra, with graded dimension $2^{n}$.

\section{Review of some geometric combinatorics}

We begin with definitions of graph associahedra; the reader is encouraged to see [4, Sect. 1] and [6] for further details.

Definition 2.1 Let $G$ be a finite connected simple graph. A tube is a set of nodes of $G$ whose induced graph is a connected subgraph of $G$. Two tubes $u$ and $v$ may interact on the graph as follows:

(1) Tubes are nested if $u \subset v$.

(2) Tubes are far apart if $u \cup v$ is not a tube in $G$, that is, the induced subgraph of the union is not connected, or none of the nodes of $u$ are adjacent to a node of $v$.

Tubes are compatible if they are either nested or far apart. We call $G$ itself the universal tube. A tubing $U$ of $G$ is a set of tubes of $G$ such that every pair of tubes in $U$ is compatible; moreover, we force every tubing of $G$ to contain (by default) its universal tube. By the term $k$-tubing we refer to a tubing made up of $k$ tubes, for $k \in\{1, \ldots, n\}$.

When $G$ is a disconnected graph with connected components $G_{1}, \ldots, G_{k}$, an additional condition is needed: If $u_{i}$ is the tube of $G$ whose induced graph is $G_{i}$, then any tubing of $G$ cannot contain all of the tubes $\left\{u_{1}, \ldots, u_{k}\right\}$. However, the universal tube is still included despite being disconnected. Parts (a)-(c) of Fig. 3 from [6] show examples of allowable tubings, whereas (d)-(f) depict the forbidden ones. 


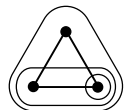

(a)

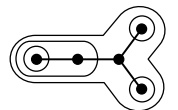

( b )

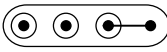

(c)

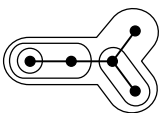

(d)

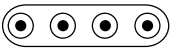

(e )

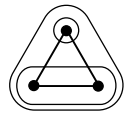

(f)

Fig. 3 (a)-(c) Allowable tubings and (d)-(f) forbidden tubings, figure from [6]
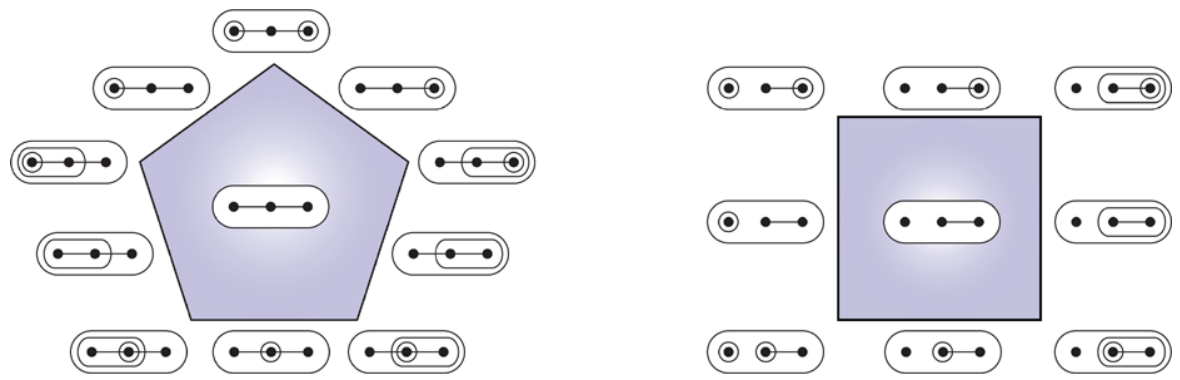

Fig. 4 Graph associahedra of a path and a disconnected graph. The 3-cube is found as the graph associahedron of two disjoint edges on four nodes, but no simple graph yields the 4-cube

Theorem 2.2 [4, Sect. 3] For a graph $G$ with $n$ nodes, the graph associahedron $\mathcal{K} G$ is a simple, convex polytope of dimension $n-1$ whose face poset is isomorphic to the set of tubings of $G$, ordered by the relationship $U \prec U^{\prime}$ if $U$ is obtained from $U^{\prime}$ by adding tubes.

The vertices of the graph associahedron are the $n$-tubings of $G$. Faces of dimension $k$ are indexed by $(n-k)$-tubings of $G$. In fact, the barycentric subdivision of $\mathcal{K} G$ is precisely the geometric realization of the described poset of tubings. Many of the face vectors of graph associahedra for path-like graphs have been found, as shown in [16]. This source also contains the face vectors for the cyclohedra. There are many open questions regarding formulas for the face vectors of graph associahedra for specific types of graphs.

Example 2.3 Figure 4, partly from [6], shows two examples of graph associahedra. These have underlying graphs a path and a disconnected graph, respectively, with three nodes each. These turn out to be the 2-dimensional associahedron and a square. The case of a three node complete graph, which is both the cyclohedron and the permutohedron, is shown in Fig. 2.

To describe the face structure of the graph associahedra we need a definition from [4, Sect. 2].

Definition 2.4 For graph $G$ and a collection of nodes $t$, construct a new graph $G^{*}(t)$ called the reconnected complement: If $V$ is the set of nodes of $G$, then $V-t$ is the set of nodes of $G^{*}(t)$. There is an edge between nodes $a$ and $b$ in $G^{*}(t)$ if $\{a, b\} \cup t^{\prime}$ is connected in $G$ for some $t^{\prime} \subseteq t$. 

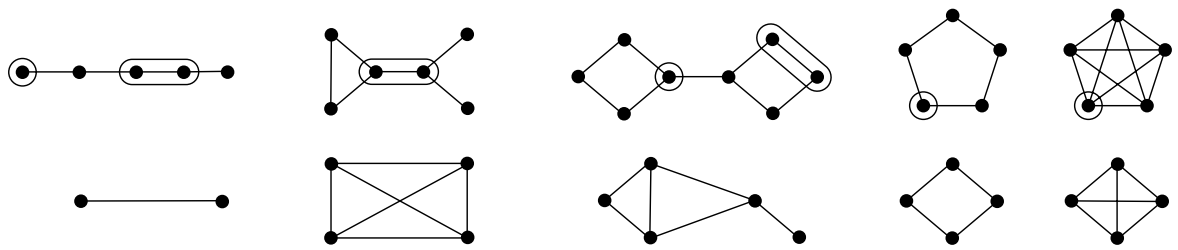

Fig. 5 Examples of tubes and their reconnected complements

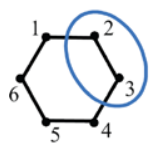

$\mathcal{K}_{2} \times \mathcal{W}_{4}$
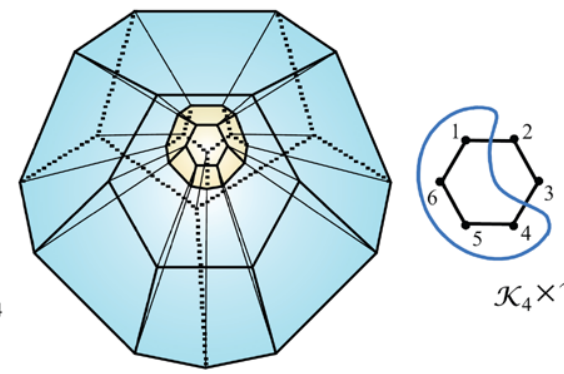

$\mathcal{K}_{4} \times \mathcal{W}_{2}$

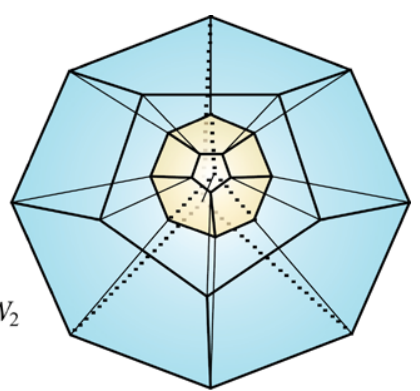

Fig. 6 Two facets of the cyclohedron $\mathcal{W}_{6}$

Example 2.5 Figure 5 illustrates some examples of graphs along with their reconnected complements.

For a given tube $t$ and a graph $G$, let $G(t)$ denote the induced subgraph on the graph $G$. By abuse of notation, we sometimes refer to $G(t)$ as a tube.

Theorem 2.6 [4, Theorem 2.9] Let $V$ be a facet of $\mathcal{K} G$, that is, a face of dimension $n-2$ of $\mathcal{K} G$, where $G$ has $n$ nodes. Let $t$ be the single, non-universal, tube of $V$. The face poset of $V$ is isomorphic to $\mathcal{K} G(t) \times \mathcal{K} G^{*}(t)$.

A pair of examples is shown in Fig. 6. The isomorphism described in [4, Theorem 2.9] is called $\hat{\rho}$. Since we will be using this isomorphism more than once as an embedding of faces in polytopes, then we will specify it, according to the tube involved, as $\hat{\rho}_{t}: \mathcal{K} G(t) \times \mathcal{K} G^{*}(t) \hookrightarrow \mathcal{K} G$.

Given a pair of tubings from $T \in \mathcal{K} G_{t}$ and $T^{\prime} \in \mathcal{K} G^{*}(t)$ the image $\hat{\rho}\left(T, T^{\prime}\right)$ consists of all of $T$ and an expanded version of $T^{\prime}$. In the latter each tube of $T^{\prime}$ is expanded by taking its union with $t$ if that union is itself a tube. A specific example of the action of $\hat{\rho}_{t}$ is in Fig. 7. In fact, often there will be more than one tube involved, as we now indicate:

Corollary 2.7 Let $\left\{t_{1}, \ldots, t_{k}, G\right\}$ be an explicit tubing of $G$, such that each pair of non-universal tubes in the list is far apart. Then the face of $\mathcal{K} G$ associated to that tubing is isomorphic to $\mathcal{K} G\left(t_{1}\right) \times \cdots \times \mathcal{K} G\left(t_{n}\right) \times \mathcal{K} G^{*}\left(t_{1} \cup \cdots \cup t_{k}\right)$. 

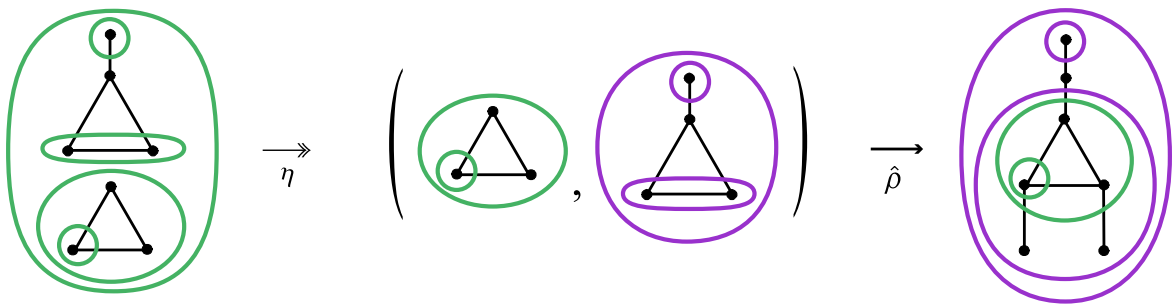

Fig. 7 Example of the action of $\eta$ and $\hat{\rho}_{t}$, where $t$ is the cycle subgraph of the final graph

We will denote the embedding by:

$$
\hat{\rho}_{t_{1} \ldots t_{k}}: \mathcal{K} G\left(t_{1}\right) \times \cdots \times \mathcal{K} G\left(t_{n}\right) \times \mathcal{K} G^{*}\left(t_{1} \cup \cdots \cup t_{k}\right) \hookrightarrow \mathcal{K} G
$$

Proof This follows directly from Theorem 2.6. Note that the reconnected complement with respect to the union of several tubes $t_{1}, \ldots, t_{k}$ is the same as taking successive iterated reconnected complements with respect to each tube in the list. That is,

$$
G^{*}\left(t_{1} \cup \cdots \cup t_{k}\right)=\left(\ldots\left(\left(G^{*}\left(t_{1}\right)\right)^{*}\left(t_{2}\right)\right)^{*} \ldots\right)^{*}\left(t_{k}\right) .
$$

Remark 2.8 As shown in [8], the graph associahedron of the graph consisting of $n$ nodes and no edges is the $(n-1)$-simplex $\Delta^{(n-1)}$. Thus the graph associahedron of a graph with connected components $G_{1}, G_{2}, \ldots, G_{k}$ is actually equivalent to the polytope $\mathcal{K} G_{1} \times \cdots \times \mathcal{K} G_{k} \times \Delta^{(k-1)}$.

This equivalence implies that:

Lemma 2.9 For a disconnected graph $G$ with multiple connected components $G_{1}, G_{2}, \ldots, G_{k}$, there is always a cellular surjection

$$
\eta: \mathcal{K} G \rightarrow \mathcal{K} G_{1} \times \cdots \times \mathcal{K} G_{k}
$$

An example is in Fig. 7.

\section{Factoring and extending the Tonks projection}

\subsection{Loday and Ronco's Hopf algebra map}

The two most important existing mathematical structures we will use in this paper are the graded Hopf algebra of permutations, $\mathfrak{S} S y m$, and the graded Hopf algebra of binary trees, $\mathcal{Y}$ Sym. The $n$th component of $\mathfrak{S} S y m$ has basis the symmetric group $S_{n}$, with number of elements counted by $n$ !. The $n$th component of $\mathcal{Y} S y m$ has basis the collection of binary trees with $n$ interior nodes, and thus $n+1$ leaves, denoted $\mathcal{Y}_{n}$. These are counted by the Catalan numbers.

The connection discovered by Loday and Ronco between the two algebras is due to the fact that a permutation on $n$ elements can be pictured as a binary tree with $n$ 

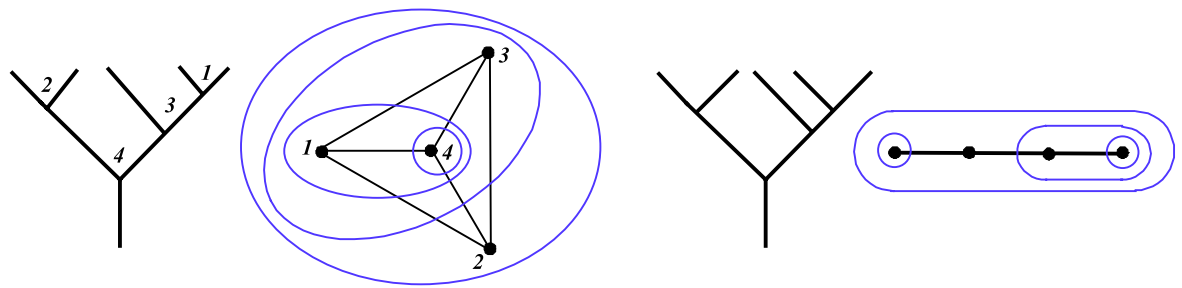

Fig. 8 The permutation $\sigma=(2431) \in S_{4}$ pictured as an ordered tree and as a tubing of the complete graph; the unordered binary tree, and its corresponding tubing
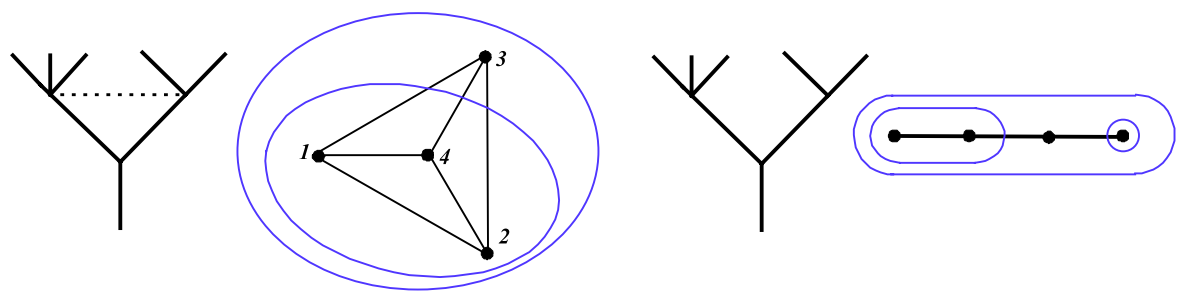

Fig. 9 The ordered partition $(\{1,2,4\},\{3\})$ pictured as a leveled tree and as a tubing of the complete graph; the underlying tree, and its corresponding tubing

interior nodes, drawn so that the interior nodes are at $n$ different vertical heights from the base of the tree. This is called an ordered binary tree. The interior nodes are numbered left to right. We number the leaves $0,1, \ldots, n-1$ and the nodes $1,2, \ldots, n-1$. The $i$ th node is "between" leaf $i-1$ and leaf $i$ where "between" might be described to mean that a rain drop falling between those leaves would be caught at that node. Distinct vertical levels of the nodes are numbered top to bottom. Then for a permutation $\sigma \in S_{n}$ the corresponding tree has node $i$ at level $\sigma(i)$. The map from permutations to binary trees is achieved by forgetting the levels, and just retaining the tree. This classical surjection is denoted $\tau: S_{n} \rightarrow \mathcal{Y}_{n}$. An example is in Fig. 8.

By a cellular surjection of polytopes $P$ and $Q$ we refer to a map $f$ from the face poset of $P$ to that of $Q$ which is onto and which preserves the poset structure. That is, if $x$ is a sub-face of $y$ in $P$ then $f(x)$ is a sub-face of or equal to $f(y)$. A cellular projection is a cellular surjection which also has the property that the dimension of $f(x)$ is less than or equal to the dimension of $x$. In [20] Tonks extended $\tau$ to a cellular projection from the permutohedron to the associahedron: $\Theta$ : $\mathcal{P}_{n} \rightarrow \mathcal{K}_{n}$. In the cellular projection a face of the permutohedron, which is leveled tree, is taken to its underlying tree, which is a face of the associahedron. Figure 9 shows an example. The new revelation of Loday and Ronco is that the map $\tau$ gives rise to a Hopf algebraic projection $\tau: \mathfrak{S S y m} \rightarrow \mathcal{Y} S y m$, so that the algebra of binary trees is seen to be embedded in the algebra of permutations.

\subsection{Tubings, permutations, and trees}

Our new approach to the Tonks projection is made possible by the recent discovery of Devadoss in [8] that the graph associahedron of the complete graph on $n$ vertices is 
precisely the $n$th permutohedron $\mathcal{P}_{n}$. Each of its vertices corresponds to a permutation of $n$ elements. Its faces in general correspond to ordered partitions of $[n]$. Keep in mind that for a permutation $\sigma \in S_{n}$, the corresponding ordered partition of $[n]$ is $\left(\left\{\sigma^{-1}(1)\right\},\left\{\sigma^{-1}(2)\right\}, \ldots,\left\{\sigma^{-1}(n)\right\}\right)$.

Here is how to describe a bijection from the $n$-tubings on the complete graph to the permutations, as found by Devadoss in [8]. First a numbering of the $n$ nodes must be chosen. Given an $n$-tubing, since the tubes are all nested we can number them starting with the innermost tube. Then the permutation $\sigma \in S_{n}$ pictured by our $n$ tubing is such that node $i$ is within tube $\sigma(i)$ but not within any tube nested inside of tube $\sigma(i)$. Figure 8 shows an example.

It is easy to extend this bijection between $n$-tubings and permutations to all tubings of the complete graph and ordered partitions of $[n]$. Given a $k$-tubing of the complete graph, each tube contains some numbered nodes which are not contained in any other tube. These subsets of $[n]$, one for each tube, make up the $k$-partition, and the ordering of the partition is from innermost to outermost. Recall that an ordered $k$-partition of $[n]$ corresponds to a leveled tree with $n+1$ leaves and $k$ levels, numbered top to bottom, at which lie the internal nodes. Numbering the $n$ spaces between leaves from left to right (imagine a raindrop falling into each space), we see that the raindrops collecting at the internal nodes at level $i$ represent the $i$ th subset in the partition. We denote our bijection by:

$$
f:\{\text { leveled trees with } n \text { leaves }\} \rightarrow \mathcal{K} \text { (complete graph on } n-1 \text { nodes }) .
$$

Figure 9 shows an example.

The binary trees with $n+1$ leaves (and $n$ internal nodes) correspond to the vertices of the $(n-1)$-dimensional associahedron, or Stasheff polytope $\mathcal{K}_{n}$. In the world of graph associahedra, these vertices correspond to the $n$-tubings of the path graph on $n$ nodes. Carr and Devadoss realized that in fact the path graph associahedron is precisely the Stasheff associahedron [4]. Thus for any tree with $n+1$ leaves we can bijectively determine a tubing on the path graph. This correspondence can be described by creating a tube of the path graph for each internal node of the tree. We number the leaves from left to right $0,1, \ldots, n$ and the nodes of the path from left to right $1, \ldots, n$. The tube we create contains the same numbered nodes of the path graph as all but the leftmost leaf of the subtree determined by the internal node. This bijection we denote by:

$$
g:\{\text { trees with } n \text { leaves }\} \rightarrow \mathcal{K} \text { (path on } n-1 \text { nodes }) .
$$

Figures 8 and 9 show examples.

\subsection{Generalizing the Tonks projection}

The fact that every graph of $n$ nodes is a subgraph of the complete graph leads us to a grand factorization of the Tonks cellular projection through all connected graph associahedra. Incomplete graphs are formed simply by removing edges from the complete graph. As a single edge is deleted, the tubing is preserved up to connection. That is, if the nodes of a tube are no longer connected, it becomes two tubes made up of the two connected subgraphs spanned by its original set of nodes. 


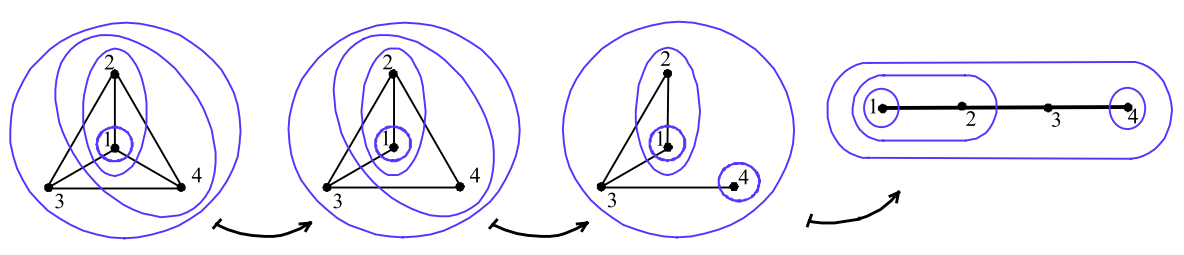

Fig. 10 The Tonks projection performed on (1243), factored by graphs

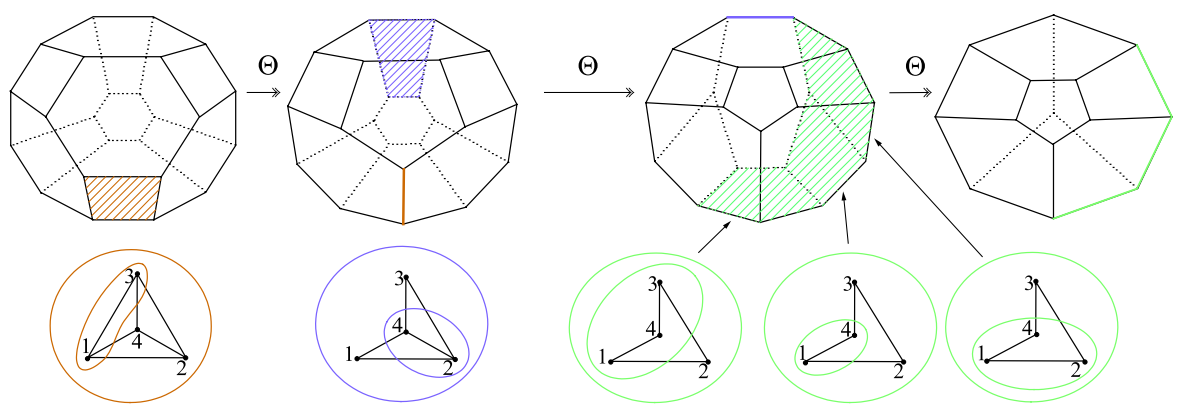

Fig. 11 A factorization of the Tonks projection through 3-dimensional graph associahedra. The shaded facets correspond to the shown tubings, and are collapsed as indicated to respective edges. The first, third and fourth pictured polytopes are above views of $\mathcal{P}_{4}, \mathcal{W}_{4}$ and $\mathcal{K}_{4}$ respectively

Definition 3.1 Let $G$ be a graph on $n$ nodes, and let $e$ be an edge of $G$. Let $G-e$ denote the graph achieved by the deletion of $e$, while retaining all $n$ nodes. We define a cellular projection $\Theta_{e}: \mathcal{K} G \rightarrow \mathcal{K}(G-e)$. First, allowing an abuse of notation, we define $\Theta_{e}$ on individual tubes. For $t$ a tube of $G$ such that $t$ is not a tube of $G-e$, then let $t^{\prime}, t^{\prime \prime}$ be the tubes of $G-e$ such that $t^{\prime} \cup t^{\prime \prime}=t$. Then:

$$
\Theta_{e}(t)= \begin{cases}\{t\}, & t \text { a tube of } G-e, \\ \left\{t^{\prime}, t^{\prime \prime}\right\}, & \text { otherwise }\end{cases}
$$

Now given a tubing $T$ on $G$ we define its image as follows:

$$
\Theta_{e}(T)=\bigcup_{t \in T} \Theta_{e}(t) .
$$

See Figs. 10, 11 and 12 for examples.

Lemma 3.2 For a graph $G$ with an edge $e, \Theta_{e}$ is a cellular surjection of polytopes $\mathcal{K} G \rightarrow \mathcal{K}(G-e)$.

Proof By Theorem 2.2 we have that the face posets of the polytopes are isomorphic to the posets of tubings. The map takes a tubing on $G$ to a tubing on $G-e$ with a greater or equal number of tubes. This establishes its projective property. Also, for two tubings $U \prec U^{\prime}$ of $G$ we see that either $\Theta_{e}(U)=\Theta_{e}\left(U^{\prime}\right)$ or $\Theta_{e}(U) \prec \Theta_{e}\left(U^{\prime}\right)$. Thus the poset structure is preserved by the projection. 


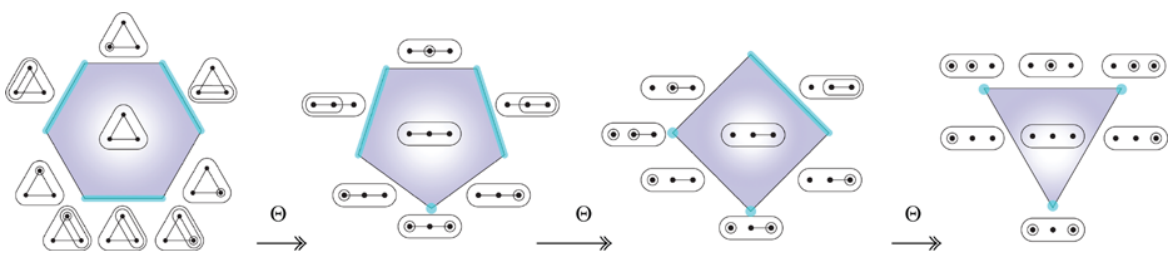

Fig. 12 Extending the Tonks projection to the 2-simplex. The highlighted edges are collapsed to the respective vertices

Finally, the map is surjective, since given any tubing $T$ on $G-e$ we can find a (maximal) preimage $T^{\prime}$ as follows: First consider all the tubes of $T$ as a candidate tubing of $G$. If it is a valid tubing, we have our $T^{\prime}$. If not, then there must be a pair of tubes $t^{\prime}, t^{\prime \prime} \in T$ which are adjacent via the edge $e$, and for which there are no tubes containing one of $t^{\prime}, t^{\prime \prime}$ but not the other. Then let $T_{1}$ be the result of replacing that pair in $T$ with the single tube $t=t^{\prime} \sqcup t^{\prime \prime}$. If $T_{1}$ is a valid tubing of $G$ then let $T^{\prime}=T_{1}$. If not, continue inductively.

Composition of these cellular projections is commutative.

Lemma 3.3 Let $e, e^{\prime}$ be edges of $G$. Then $\Theta_{e} \circ \Theta_{e^{\prime}}=\Theta_{e^{\prime}} \circ \Theta_{e}$.

Proof Consider the image of a tubing of $G$ under either composition. A tube of $G$ that is a tube of both $G-e$ and $G-e^{\prime}$ will persist in the image. Otherwise it will be broken, perhaps twice. The same smaller tubes will result regardless of the order of the breaking.

By Lemma 3.3 we can unambiguously use the following notation:

Definition 3.4 For any collection $E$ of edges of $G$ we denote by $\Theta_{E}: \mathcal{K} G \rightarrow$ $\mathcal{K}(G-E)$ the composition of projections $\left\{\Theta_{e} \mid e \in E\right\}$.

Now the Tonks projection from leveled trees to trees can be described in terms of the tubings. Beginning with a tubing on the complete graph with numbered nodes, we achieve one on the path graph by deleting all the edges of the complete graph except for those connecting the nodes in consecutive order from 1 to $n$. See Figs. 8, 9 and 10 for a picture to accompany the following:

Theorem 3.5 Let $e_{i, k}$ be the edge between the nodes $i, i+k$ of the complete graph $G$ on $n$ nodes. Let $P=\left\{e_{i, k} \mid i \in\{1, \ldots, n-2\}\right.$ and $\left.k \in\{2, \ldots, n-i\}\right\}$. These are all but the edges of the path graph. Then the following composition gives the Tonks map:

$$
g^{-1} \circ \Theta_{P} \circ f=\Theta
$$

Proof We begin with an ordered $j$-partition of $n$ drawn as a leveled tree $t$ with $n+1$ leaves numbered left to right, and $j$ levels numbered top to bottom. The bijection $f$ tells us how to draw $t$ as a tubing of the complete graph $K_{n}$ with numbered nodes. 
Consider the internal nodes of $t$ at level $i$. In $f(t)$ there corresponds a tube $u_{i}$ containing the nodes of $K_{n}$ with the same numbers as all the spaces between leaves of the subtrees rooted at the internal nodes at level $i$. The set in the partition which is represented by the internal nodes at level $i$ is the precise set of nodes of $u_{i}$ which are not contained by any other tube in $f(t)$. The relative position of this subset of $[n]$ in our ordered partition is reflected by the relative nesting of the tube $u_{i}$.

The map $\Theta_{P}$ has the following action on tubings. Let $u$ be a tube of $f(t)$. We partition $u$ into subsets of consecutively numbered nodes such that no union of two subsets is consecutively numbered. Then the tubing $\Theta_{P}(f(t))$ contains the tubes given by these subsets.

Now we claim that the tree $g^{-1}\left(\Theta_{P}(f(t))\right)$ has the same branching structure as $t$ itself. First, for any interior node of $t$ there is a tube of consecutively numbered nodes of the path graph, arising from the original tube of $f(t)$. This then becomes a corresponding interior node of our tree, under the action of $g^{-1}$. Secondly, if any interior node of $t$ lies between the root of $t$ and a second interior node, then the same relation holds in the tree between the corresponding interior nodes. This follows from the fact that the action of any of our maps $\Theta_{e_{i, k}}$ will preserve relative nesting. That is, for two tubes $u \subset v$ we have that in the image of $\Theta_{e_{i, k}}$ any tube resulting from $u$ must lie within a tube resulting from $v$.

To sum up, there is a factorization of the Tonks cellular projection through various graph associahedra. An example on an $n$-tubing is shown in Fig. 10, and another possible factorization of the projection in dimension 3 is demonstrated in Fig. 11.

\subsection{Disconnected graph associahedra}

The special case of extending the Tonks projection to graphs with multiple connected components will be useful. Consider a partition $S_{1} \sqcup \cdots \sqcup S_{k}$ of the $n$ nodes of a connected graph $G$, chosen such that we have connected induced subgraphs $G\left(S_{i}\right)$. Let $E_{S}$ be the set of edges of $G$ not included in any $G\left(S_{i}\right)$. Thus the graph $G-E_{S}$ formed by deleting these edges will have the $k$ connected components $G\left(S_{i}\right)$. In this situation $\Theta_{E_{S}}$ will be a generalization of the Tonks projection to the graph associahedron of a disconnected graph.

In Fig. 12 we show the extended Tonks projections in dimension 2.

\section{Geometrical view of $\mathfrak{S S y m}$ and $\mathfrak{Y} S y m$}

Before proving the graded algebra structures on graph associahedra which our title promised, we motivate our point of view by showing how it will fit with the well known graded algebra structures on permutations and binary trees.

\subsection{Review of SSym}

Let $\mathfrak{S} S y m$ be the graded vector space over $\mathbb{Q}$ with the $n$th component of its basis given by the permutations $S_{n}$. An element $\sigma \in S_{n}$ is given by its image $(\sigma(1), \ldots, \sigma(n))$, 
often without commas. We follow [1] and [2] and write $F_{u}$ for the basis element corresponding to $u \in S_{n}$ and 1 for the basis element of degree 0. A graded Hopf algebra structure on SSym was discovered by Malvenuto and Reutenauer in [13]. First we review the product and coproduct and then show a new way to picture those operations.

Recall that a permutation $\sigma$ is said to have a descent at location $p$ if $\sigma(p)>$ $\sigma(p+1)$. The $(p, q)$-shuffles of $S_{p+q}$ are the $(p+q)$ permutations with at most one descent, at position $p$. We denote this set as $S^{(p, q)}$. The product in SSym of two basis elements $F_{u}$ and $F_{v}$ for $u \in S_{p}$ and $v \in S_{q}$ is found by summing a term for each shuffle, created by composing the juxtaposition of $u$ and $v$ with the inverse shuffle:

$$
F_{u} \cdot F_{v}=\sum_{\iota \in S^{(p, q)}} F_{(u \times v) \cdot \iota^{-1}} .
$$

Here $u \times v$ is the permutation $(u(1), \ldots, u(p), v(1)+p, \ldots, v(q)+p)$.

\subsection{Geometry of $\mathfrak{S S y m}$}

The algebraic structure of $\mathfrak{S} S y m$ can be linked explicitly to the recursive geometric structure of the permutohedra. In $\mathfrak{S} S y m$ we may view our operands (a pair of permutations) as a vertex of the cartesian product of permutohedra $\mathcal{P}_{p} \times \mathcal{P}_{q}$. Then their product is the formal sum of images of that vertex under the collection of inclusions of $\mathcal{P}_{p} \times \mathcal{P}_{q}$ as a facet of $\mathcal{P}_{p+q}$. An example is in Fig. 13, where the product is shown along with corresponding pictures of the tubings on the complete graphs. To make this geometric claim precise we use the facet isomorphism $\hat{\rho}_{t}$ which exists by Theorem 2.6.

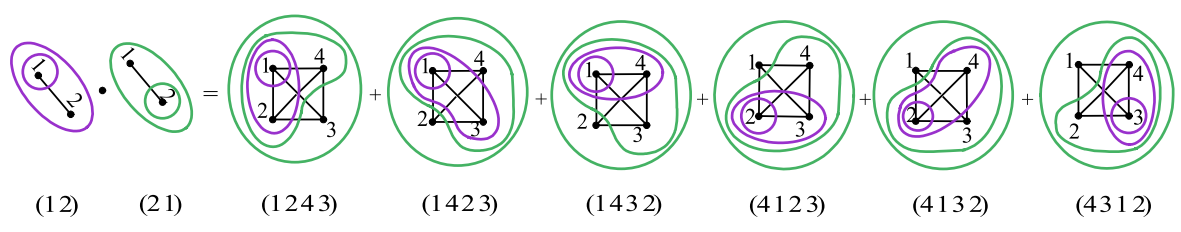

(1 2 )

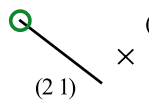

$\mathcal{P}_{2}$
(1 2$)$

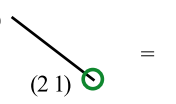

$\mathcal{P}_{2}$

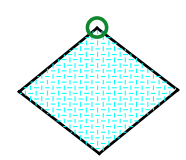

$\mathcal{P}_{2} \times \mathcal{P}_{2}$

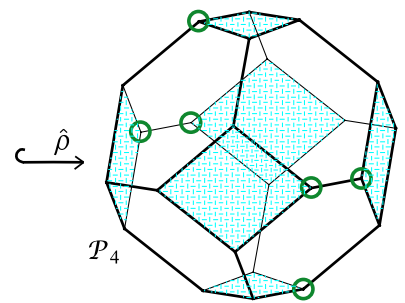

Fig. 13 The product in SSym. Here (and similarly in all our pictorial examples) we let the picture of the graph tubing $u$ stand in for the basis element $F_{u}$. In the picture of $\mathcal{P}_{4}$ the circled vertices from bottom to top are the permutations in the product, as listed from left to right above 
Theorem 4.1 The product in SSym of basis elements $F_{u}$ and $F_{v}$ for $u \in S_{p}$ and $v \in S_{q}$ may be written:

$$
F_{u} \cdot F_{v}=\sum_{\iota \in S^{(p, q)}} F_{\hat{\rho}_{\iota}(u, v)}
$$

where $\hat{\rho}_{\iota}$ is shorthand for $\hat{\rho}_{l([p])}$.

Proof From Theorem 2.6 we have that for each tube $t$ of the graph, the corresponding facet of $\mathcal{K} G$ is isomorphic to $\mathcal{K} G^{*}(t) \times \mathcal{K} G(t)$. In the case of the complete graph $G=$ $K_{p+q}$, for any tube $t$ of $p$ nodes, we have the facet inclusion $\hat{\rho}_{t}: \mathcal{P}_{q} \times \mathcal{P}_{p} \rightarrow \mathcal{P}_{p+q}$.

We just need to review the definition of the isomorphism $\hat{\rho}$ from the proof of $[4$, Theorem 2.9], and point out that the permutation associated to the tubing $\hat{\rho}_{l}(u, v)$ is indeed $(u \times v) \cdot \iota^{-1}$.

Given a shuffle $\iota$, the $(p+q)$-tubing $\hat{\rho}_{\iota}(u, v)$ on $K_{p+q}$ is given by including for each tube $t$ of $u$ the tube with nodes the image $\iota(t)$. Denote the resulting set of tubes by $\overline{\imath(u)}$. For each tube $s$ of $v$ we include the tube formed by $\iota([p]) \cup \hat{\imath}(s)$, where $\hat{\imath}(i)=\iota(i+p)$. We denote the resulting set of tubes as $\bar{\imath}(v)$.

Now the tubing $\hat{\rho}_{l}(u, v)$ is defined to be $\overline{\iota(u)} \cup \overline{\hat{\imath}(v)}$. This tubing is precisely the complete tubing which represents the permutation $(u \times v) \cdot \iota^{-1}$.

To sum up, in our view of $\mathfrak{5 S y m}$ each permutation is pictured as a tubing of a complete graph with numbered nodes. Since any subset of the nodes of a complete graph spans a smaller complete graph, we can draw the terms of the product in SSym directly. Choosing a $(p, q)$-shuffle is accomplished by choosing $p$ nodes of the $(p+q)$-node complete graph $K_{p+q}$. First the permutation $u$ (as a $p$-tubing) is drawn upon the induced $p$-node complete subgraph, according to the ascending order of the chosen nodes. Then the permutation $v$ is drawn upon the subgraph induced by the remaining $q$ nodes-with a caveat. Each of the tubes of $v$ is expanded to also include the $p$ nodes that were originally chosen. This perspective will generalize nicely to the other graphs and their graph associahedra.

In [1] and [2] the authors give a related geometric interpretation of the products of SSym and $\mathcal{Y}$ Sym as expressed in the Möbius basis. An interesting project for the future would be to apply that point of view to $\mathcal{W} S y m$.

Remark 4.2 The coproduct of SSym can also be described geometrically in terms of the graph tubings. The coproduct is usually described as a sum of all the ways of splitting a permutation $u \in S_{n}$ into two permutations $u_{i} \in S_{i}$ and $u_{n-i} \in S_{n-i}$ :

$$
\Delta\left(F_{u}\right)=\sum_{i=0}^{n} F_{u_{i}} \otimes F_{u_{n-i}}
$$

where $u_{i}=(u(1) \ldots u(i))$ and $u_{n-i}=(u(i+1)-i \ldots u(n)-i)$.

Given an $n$-tubing $u$ of the complete graph on $n$ vertices we can find $u_{i}$ and $u_{n-i}$ just by restricting to the subgraphs (also complete) induced by the nodes $1, \ldots, i$ and $i+1, \ldots, n$ respectively. For each tube $t \in u$ the two intersections of $t$ with the 


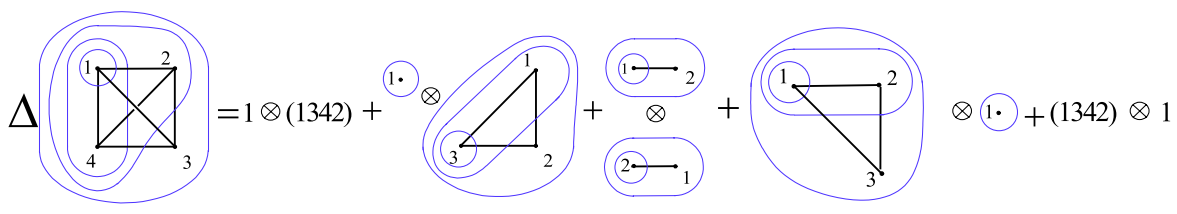

Fig. 14 The coproduct in $\mathfrak{S} S y m$

respective subgraphs are included in the respective tubings $u_{i}$ and $u_{n-i}$. An example is in Fig. 14.

Notice that this restriction of the tubings to subgraphs is the same as the result of performing the Tonks projection. Technically, $\left(u_{i}, u_{n-i}\right)=\eta\left(\Theta_{E_{i}}(u)\right)$, where

$$
E_{i}=\left\{\text { e an edge of } \mathrm{G} \mid e \text { connects a node } j \leq i \text { to a node } j^{\prime}>i\right\},
$$

and $\eta$ is from Lemma 2.9 .

$$
\Delta F_{(1342)}=1 \otimes F_{(1342)}+F_{(1)} \otimes F_{(231)}+F_{(12)} \otimes F_{(21)}+F_{(123)} \otimes F_{(1)}+F_{(1342)} \otimes 1 .
$$

\subsection{Review of $\mathcal{Y} S y m$}

The product and coproduct of $\mathcal{Y}$ Sym are described by Aguiar and Sottile in terms of splitting and grafting binary trees [2]. We can vertically split a tree into smaller trees at each leaf from top to bottom-as if a lightning strike hits a leaf and splits the tree along the path to the root. We graft trees by attaching roots to leaves (without creating a new interior node at the graft.) The product of two trees with $n$ and $m$ interior nodes (in that order) is a sum of $\left(\begin{array}{c}n+m \\ n\end{array}\right)$ terms, each with $n+m$ interior nodes. Each is achieved by vertically splitting the first tree into $m+1$ smaller trees and then grafting them to the second tree, left to right. A picture is in Fig. 15.

\subsection{Geometry of $\mathcal{Y}$ Sym}

Since Loday and Ronco demonstrated that the Tonks projection, restricted to vertices, gives rise to an algebra homomorphism $\boldsymbol{\tau}: \mathfrak{S S y m} \rightarrow \mathcal{Y}$ Sym, it is no surprise that the processes of splitting and grafting have geometric interpretations. Grafting corresponds to certain face inclusions of associahedra, and splitting corresponds to the extension of Tonks's projection to disconnected graphs. To see the latter, note that splitting at leaf $i$ is the same as deleting the edge from node $i$ to node $i+1$. Thus the product in $\mathcal{Y} S y m$ can be described with the language of path graph tubings, using a combination of facet inclusion and the extended Tonks projection. An example is shown in Fig. 15.

Let $U$ be the path graph with $p$-tubing $u$ and $V$ the path graph with $q$-tubing $v$. Given a shuffle $\iota \in S^{(p, q)}$ we can partition the nodes of $U$ into the preimages $s_{1}, \ldots, s_{k}$ of the connected components $\iota\left(s_{1}\right), \ldots, \iota\left(s_{k}\right)$ of the possibly disconnected subgraph induced by the nodes $\iota([p])$ on the $(p+q)$-path. Let $E_{\iota}$ be the edges of $U$ not included in the subgraphs $U\left(s_{i}\right)$ induced by our partition. For short we denote 


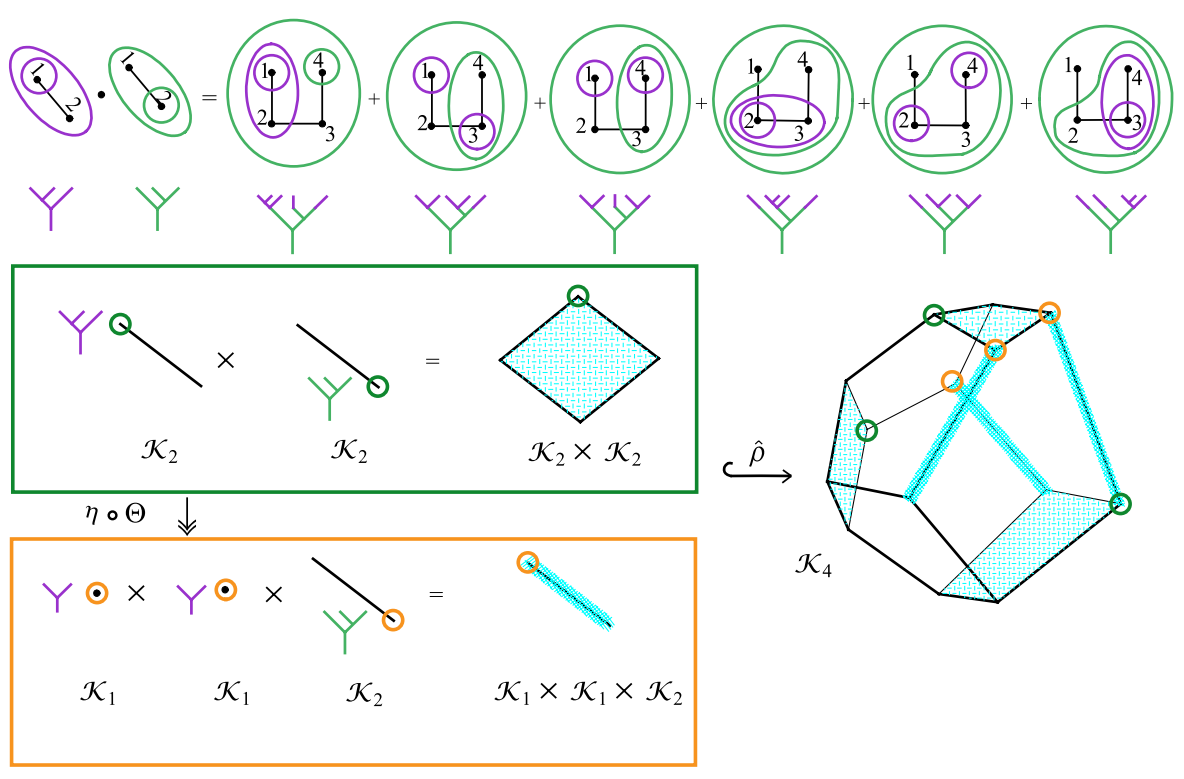

Fig. 15 The product in $\mathcal{Y}$ Sym. The circled vertices of $\mathcal{K}_{4}$ which are at the upper end of highlighted edges are the fifth, third and second terms of the product, in that order respectively from bottom to top in the picture

the extended Tonks projection $\Theta_{E_{l}}$ as simply $\Theta_{\iota}$. Now $\Theta_{\iota}(u)$ is the projection of $u$ onto the possibly disconnected graph $U-E_{\iota}$. Recall from Lemma 2.9 that a vertex of $\mathcal{K}\left(U-E_{\iota}\right)$ may be mapped to a vertex of $\mathcal{K} U\left(s_{1}\right) \times \cdots \times \mathcal{K} U\left(s_{k}\right)$. We call this $\operatorname{map} \eta_{\iota}$.

Theorem 4.3 The product in YSym can be written:

$$
F_{u} \cdot F_{v}=\sum_{\iota \in S^{(p, q)}} F_{\hat{\rho}_{\iota}\left(\eta_{\iota}\left(\Theta_{\iota}(u)\right), v\right)},
$$

where $\hat{\rho}_{\iota}$ is shorthand notation for the isomorphism $\hat{\rho}_{l\left(s_{1}\right) \ldots l\left(s_{k}\right)}$ from Corollary 2.7.

Proof We will explain how the splitting and grafting of trees in a term of the product may be put into the language of tubings, and then argue that the term thus described is indeed the image of projections and inclusions as claimed. The product in $\mathcal{Y} S y m$ of two basis elements $F_{u}$ and $F_{v}$ for $u \in \mathcal{Y}_{p}$ and $v \in \mathcal{Y}_{q}$ is found by summing a term for each shuffle $\iota \in S^{(p, q)}$. We draw $u$ and $v$ in the form of tubings on path graphs of $p$ and $q$ nodes, respectively.

Here is the non-technical description: first the $p$-tubing $u$ is drawn upon the induced subgraph of the nodes $\iota([p])$ according to the ascending order of the chosen nodes. However, each tube may need to be first broken into several sub-tubes. Then the $q$-tubing $v$ is drawn upon the subgraph induced by the remaining $q$ nodes. In this last step, each of the tubes of $v$ is expanded to also include any of the previously drawn tubes that its nodes are adjacent to. 
To be precise, we first choose a shuffle $\iota \in S^{(p, q)}$. Let $\hat{\imath}(i)=\iota(i+p)$. Our term of $F_{u} \cdot F_{v}$ is the $(p+q)$-tubing on the $(p+q)$-path given by the following:

First for each tube $t \in u$ we include in our new tubing the tubes which are the connected components of the subgraph induced by the nodes $\iota(t)$. Let $\overline{\imath(u)}$ denote the tubing constructed thus far. After this step in terms of trees, we have performed the splitting and chosen where to graft the (non-trivial) subtrees. In terms of trees the splitting occurs at leaves labeled by $\hat{\imath}(i)-i$ for $i \in[q]$.

Second, for each tube $s \in v$ we include in our term of the product the tube formed by

$$
\hat{\imath}(s) \cup\left\{j \in t^{\prime} \in \overline{\imath(u)} \mid t^{\prime} \text { is adjacent to } \hat{\imath}(s)\right\} .
$$

Let $\overline{\hat{\imath}(v)}$ denote the tubes added in this second step. Now we have completed the grafting operation.

Now we just point out that $\hat{\rho}_{l}\left(\eta_{\iota}\left(\Theta_{\iota}(u)\right), v\right)$ is precisely the same as $\overline{\iota(u)} \cup \overline{\hat{\imath}(v)}$. The splitting of tubes of $u$ is accomplished by $\Theta_{\iota}$; and $\eta_{\iota}$ simply recasts the result as an element of the appropriate cartesian product. Then $\hat{\rho}_{l}$, as defined in [4], performs the inclusion of that element paired together with $v$.

To summarize, the product in $\mathcal{Y} S y m$ can be seen as a process of splitting and grafting, or equivalently of projecting and including. From the latter viewpoint, we see the product of two associahedra vertices being achieved by projecting the first onto a cartesian product of smaller associahedra, and then mapping that result paired with the second operand into a large associahedron via face inclusion. Notice that the reason the second path graph tubing $v$ can be input here is that any reconnected complement of a path graph is another path graph.

Remark 4.4 The coproduct of $\mathcal{Y}$ Sym can also be described geometrically in terms of the graph tubings. The coproduct is usually described as a sum of all the ways of splitting a binary tree $u \in \mathcal{Y}_{n}$ along leaf $i$ into two trees: $u_{i} \in \mathcal{Y}_{i}$ and $u_{n-i} \in \mathcal{Y}_{n-i}$ :

$$
\Delta\left(F_{u}\right)=\sum_{i=0}^{n} F_{u_{i}} \otimes F_{u_{n-i}} .
$$

Given an $n$-tubing $u$ of the path graph on $n$ vertices we can find $u_{i}$ and $u_{n-i}$ just by restricting to the subgraphs (also paths) induced by the nodes $1, \ldots, i$ and $i+1, \ldots, n$ respectively. For each tube $t \in u$ the two intersections of $t$ with the respective subgraphs are included in the respective tubings $u_{i}$ and $u_{n-i}$. An example is in Fig. 16.

$\Delta$

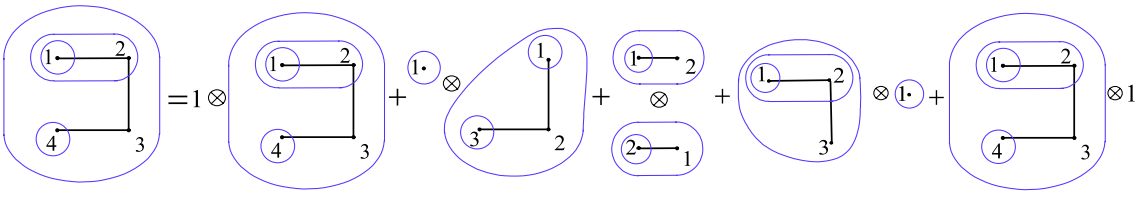

Fig. 16 The coproduct in $\mathcal{Y}$ Sym 
Notice that this restriction of the tubings to subgraphs is the same as the result of performing the extended Tonks projection, just as described in Remark 4.2.

\section{The algebra of the vertices of cyclohedra}

Recall that the $(n-1)$-dimensional cyclohedron $\mathcal{W}_{n}$ has vertices which are indexed by $n$-tubings on the cycle graph of $n$ nodes. We will define a graded algebra with a basis which corresponds to the vertices of the cyclohedra, and whose grading respects the dimension (plus one) of the cyclohedra.

Definition 5.1 Let $\mathcal{W} S y m$ be the graded vector space over $\mathbb{Q}$ with the $n$th component of its basis given by the $n$-tubings on the cycle graph of $n$ cyclically numbered nodes. By $W_{n}$ we denote the set of $n$-tubings on the cycle graph $C_{n}$. We write $F_{u}$ for the basis element corresponding to $u \in W_{n}$ and 1 for the basis element of degree 0 .

\subsection{Graded algebra structure}

Now we demonstrate a product which respects the grading on $\mathcal{W}$ Sym by following the example described above for 5 Sym. The product in $\mathcal{W} S y m$ of two basis elements $F_{u}$ and $F_{v}$ for $u \in W_{p}$ and $v \in W_{q}$ is found by summing a term for each shuffle $\iota \in S^{(p, q)}$. First the $p$-tubing $u$ is drawn upon the induced subgraph of the nodes $\iota([p])$ according to the ascending order of the chosen nodes. However, each tube may need to be first broken into several sub-tubes, since the induced graph on the nodes $\iota([p])$ may have connected components $\iota\left(s_{1}\right), \ldots, \iota\left(s_{k}\right)$ (as described in Sect. 4.4). Then the $q$-tubing $v$ is drawn upon the subgraph induced by the remaining $q$ nodes. However, each of the tubes of $v$ is expanded to also include any of the previously drawn tubes that its nodes are adjacent to.

\section{Definition 5.2}

$$
F_{u} \cdot F_{v}=\sum_{\iota \in S^{(p, q)}} F_{\hat{\rho}_{l}\left(\eta_{\iota}\left(\Theta_{\iota}(u)\right), v\right) .}
$$

Here $\hat{\rho}_{\iota}$ is shorthand notation for the isomorphism $\hat{\rho}_{l\left(s_{1}\right) \ldots \iota\left(s_{k}\right)}$ from Corollary 2.7. Also 1 is a two-sided unit.

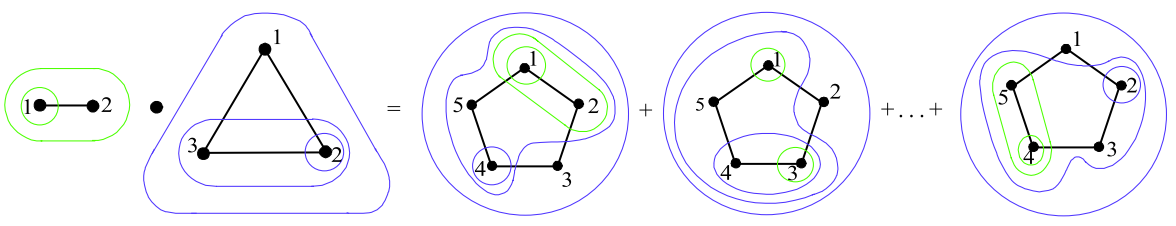

Fig. 17 A product of cycle graph tubings (10 terms total) 

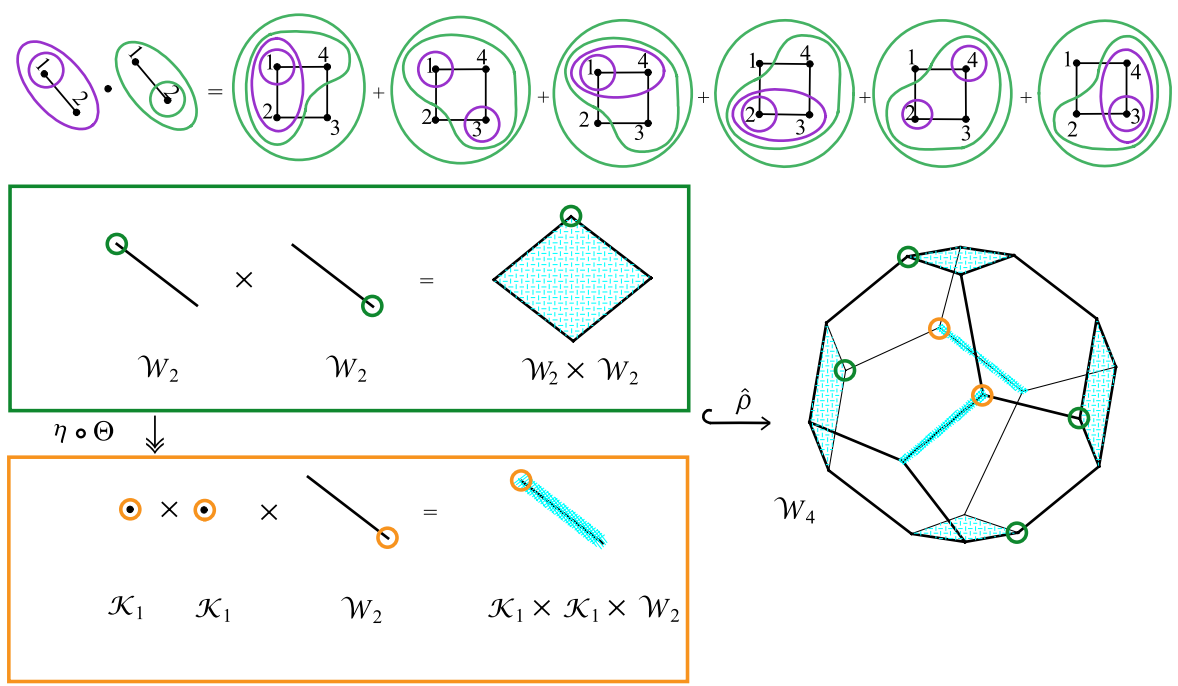

Fig. 18 The product in $\mathcal{W}$ Sym. The second and fifth terms of the product are the ones that use a Tonks projection; they are found as the two vertices at the tips of included (highlighted) edges

An example is shown in Fig. 17. For an example of finding a term in the product given a specific shuffle $\iota$, see the second part of Fig. 19 .

Theorem 5.3 The product we have just described makes $\mathcal{W}$ Sym into an associative graded algebra.

Proof Given a shuffle $\iota$ we will use the fact that

$$
\hat{\rho}_{\iota}\left(\eta_{\iota}\left(\Theta_{\iota}(u)\right), v\right)=\overline{\iota(u)} \cup \overline{\hat{\imath}(v)}
$$

as defined in the proof of Theorem 4.3. First we must check that the result of the product is indeed a sum of valid $(p+q)$-tubings of the cycle graph $C_{p+q}$. We claim that in each term the new tubes we have created are pairwise compatible. This being shown, we will be able to deduce that since $p+q$ tubes were used in the construction, then the resulting term will necessarily have $p+q$ tubes as well. To check our claim we compare pairs of tubes in one or both of $\overline{\imath(u)}$ and $\overline{\hat{\imath}(v)}$. There are six cases.

(1) By our method of construction, any tube of $\overline{\imath(u)}$ is either nested within some of or far apart from all of the tubes of $\bar{\imath}(v)$.

(2) If two tubes of $\overline{\iota(u)}$ are made up of nodes in $\iota(t)$ and $\iota\left(t^{\prime}\right)$ respectively for nested tubes $t$ and $t^{\prime}$ of $u$ then they will be similarly nested.

(3) Two tubes from $\overline{\iota(u)}$ might both be made up of nodes in $\iota(t)$ for a single tube $t$ of $u$. In that case they are guaranteed to be far apart, since their respective nodes together cannot be a consecutive string $(\bmod p+q)$.

(4) If two tubes of $\overline{\iota(u)}$ are made up of nodes in $\iota(t)$ and $\iota\left(t^{\prime}\right)$ respectively for far apart tubes $t$ and $t^{\prime}$ of $u$, then we claim they will be far apart. This is true since if two nodes $a$ and $b$ are nonadjacent in the cycle graph $C_{p}$ then the two nodes $\iota(a)$ and $\iota(b)$ will be nonadjacent in $C_{p+q}$. 


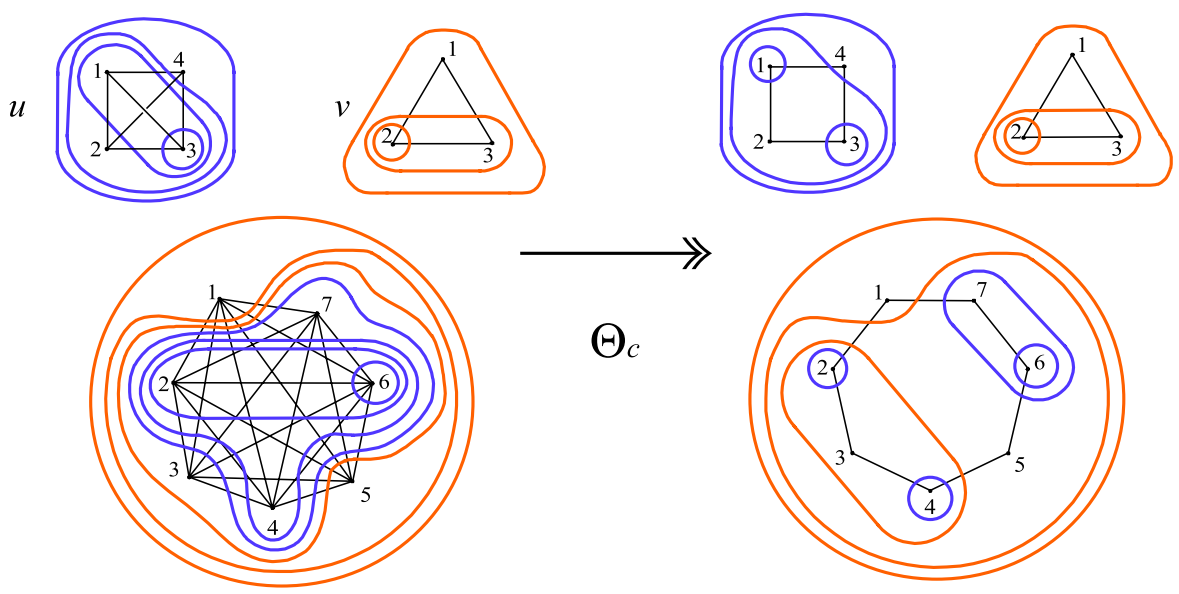

Fig. 19 An example of the equation $\Theta_{c}(\overline{\imath(u)} \cup \overline{\hat{\imath}(v)})=\overline{\imath\left(\Theta_{c}(u)\right)} \cup \overline{\hat{\imath}\left(\Theta_{c}(v)\right)}$. where $u=(2314), v=(312)$ and $\iota([4])=\{2,4,6,7\}$

(5) If two tubes of $\overline{\hat{\imath}(v)}$ contain some nodes in $\hat{\imath}(t)$ and $\hat{\imath}\left(t^{\prime}\right)$ respectively for nested tubes $t$ and $t^{\prime}$ of $u$ then they will be similarly nested. This follows from the fact that $\iota(t)$ will only be adjacent to nodes that $\iota\left(t^{\prime}\right)$ is also adjacent to.

(6) Finally, if two tubes of $\bar{\imath}(v)$ contain some nodes in $\hat{\imath}(t)$ and $\hat{\imath}\left(t^{\prime}\right)$ respectively for far apart tubes $t$ and $t^{\prime}$ of $u$, then we claim they will be far apart. This final case depends on a special property which the cycle graphs exemplify. Given any subset of $k$ of the nodes of $C_{n}$, the reconnected complement of that subset is the cycle graph $C_{n-k}$. Specifically the reconnected complement of $C_{p+q}$ with respect to the nodes $\iota(p)$ is the graph $C_{q}$. Thus even the expanded tubes of $\bar{\imath}(v)$ remain far apart as long as their components from $\hat{\imath}(q)$ were far apart; and this last property is guaranteed since $\iota$ preserves the cyclic order.

Thus we have shown that the result of multiplying two basis elements is again a basis element, and that this multiplication respects the grading. That this multiplication is associative is a corollary of the following result regarding how the multiplication is preserved under a map from $\mathfrak{S} S y m$, specifically a corollary of Theorem 5.6.

Remark 5.4 Note that the cases (1) and (2) are explainable simply by the fact that we start with two valid tubings and multiply them in the given order. Cases (3)-(6) however can be jointly explained based upon the fact that given any subset of $k$ of the nodes of $C_{n}$, the reconnected complement of that subset is the cycle graph $C_{n-k}$. Cases (3)-(5) specifically rely on the fact that the reconnected complement of $C_{p+q}$ with respect to the nodes $\hat{\imath}(q)$ is the graph $C_{p}$. This property is true of many other graph sequences, including the complete graphs and the path graphs. The property is also more simply stated as follows: the reconnected complement of $G_{i}$ with respect to any single node is $G_{i-1}$.

Remark 5.5 Once again we can interpret the product geometrically. The entire contents of the proof of Theorem 4.3 apply here, with the term "path" everywhere replaced with the term "cycle." Thus a term in the product can be seen as first projecting 
the cyclohedron vertex $u$ onto a collection of sub-path graphs of the cycle. We then map the vertex of this cartesian product of associahedra, paired with the second vertex of the cyclohedron represented by $v$, into the large cyclohedron via the indicated face inclusion. The usual picture is in Fig. 18.

\subsection{Algebra homomorphisms}

Next we consider the map from the permutohedra to the cyclohedra which is described via the deletion of edges - from the complete graphs to the cycle graphs - and point out that this is an algebra homomorphism. Recall from Theorem 3.5 that $\Theta_{P}$ is the Tonks projection viewed from the graph associahedra point of view; via deletion of the edges of the complete graph except for the path connecting the numbered nodes in order. Let $\Theta_{c}$ be the map defined just as $\Theta_{P}$ but without deleting the edge from node $n$ to node 1 . Thus we will be deleting all the edges except those making up the cycle of numbered nodes in cyclic order. Define a map from $\mathfrak{S} S y m$ to $\mathcal{W} S y m$ on basis elements by:

$$
\hat{\Theta}_{c}\left(F_{u}\right)=F_{\Theta_{c}(u)} .
$$

Theorem 5.6 The map $\hat{\Theta}_{c}$ is an algebra homomorphism from 5 Sym onto $\mathcal{W} S y m$.

Proof For $u \in S_{p}$ and $v \in S_{q}$ we compare $\hat{\Theta}_{c}\left(F_{u} \cdot F_{v}\right)$ with $\hat{\Theta}_{c}\left(F_{u}\right) \cdot \hat{\Theta}_{c}\left(F_{v}\right)$. Each of the multiplications results in a sum of $\left(\begin{array}{c}p+q \\ p\end{array}\right)$ terms. It turns out that comparing the results of the two operations can be done piecewise. Thus we check that for a given shuffle $\iota \in S^{(p, q)}$ the respective terms of our two operations agree: we claim that

$$
\Theta_{c}\left(\hat{\rho}_{l}\left(\eta_{\iota}\left(\Theta_{\iota}(u)\right), v\right)\right)=\hat{\rho}_{\iota}\left(\eta_{\iota}\left(\Theta_{\iota}\left(\Theta_{c}(u)\right)\right), \Theta_{c}(v)\right)
$$

or equivalently:

$$
\Theta_{c}(\overline{\imath(u)} \cup \overline{\hat{\imath}(v)})=\overline{\iota\left(\Theta_{c}(u)\right)} \cup \overline{\hat{\imath}\left(\Theta_{c}(v)\right)} .
$$

Here $\overline{\imath(u)}$ and $\overline{\hat{\imath}(v)}$ are as described in the proof of Theorem 4.3, and the right-hand side of the equation is using the notation of Definition 5.2. The justification is a straightforward comparison of the indicated operations on individual tubes. There are two cases.

(1) For $t$ a tube of $u$, the right-hand side first breaks $t$ into several smaller tubes by deleting certain edges of the $p$-node complete graph, then takes each of these to their image under $\iota$, breaking them again whenever they are no longer connected. The left-hand side takes $t$ to $\iota(t)$, a tube of the complete graph on $p+q$ nodes, and then breaks $\iota(t)$ into the tubes that result from deleting the specified edges of the $(p+q)$-node complete graph. In this last step, by Lemma 3.3, we can delete first those edges that also happen to lie in the complete subgraph induced by $\iota([p])$, and then the remaining specified edges. Thus we have duplicated the left-hand side and get the same final set of tubes on either side of the equation.

(2) For $s$ a tube of $v$, the right-hand side first breaks $s$ into several smaller tubes by deleting certain edges of the $q$-node complete graph, then takes each of these to 
their image under $\hat{\imath}$, expanding them to include tubes of $\overline{\imath\left(\Theta_{c}(u)\right)}$. The left-hand side takes $s$ to $\hat{\imath}(s) \cup \iota([p])$, and then breaks the result into the tubes that result from deleting the specified edges of the $(p+q)$-node complete graph. Again we can delete first those edges that also happen to lie in the complete subgraph induced by $\iota([p])$, and then the remaining specified edges, duplicating the lefthand process.

An illustration of the two sides of the equation is in Fig. 19. The surjectivity of $\hat{\Theta}_{c}$ follows from the surjectivity of the generalized Tonks projection, as shown in Lemma 3.2.

Let $C_{n}$ be the cycle graph on $n$ numbered nodes and let $w$ be the edge from node 1 to node $n$. We can define a map from $\mathcal{W}$ Sym to $\mathcal{Y} S y m$ by:

$$
\hat{\Theta}_{w}\left(F_{u}\right)=F_{\Theta_{w}(u)},
$$

for $u \in W_{n}$.

Theorem $5.7 \hat{\Theta}_{w}$ is a surjective homomorphism of graded algebras $\mathcal{W}$ Sym $\rightarrow$ $\mathcal{Y}$ Sym.

Proof In [11] it is shown that the map we call $\boldsymbol{\tau}$ is an algebra homomorphism from $\mathfrak{S}$ Sym to $\mathcal{Y}$ Sym. In the previous theorem we demonstrated that the map $\hat{\Theta}_{c}$ is a surjective algebra homomorphism from $\mathfrak{S} S y m$ to $\mathcal{W} S y m$. Now, since $\tau$ is the same map on vertices as our $\Theta_{P}$ (from Theorem 3.5), the relationship of these three is:

$$
\boldsymbol{\tau}=\hat{\Theta}_{w} \circ \hat{\Theta}_{c} .
$$

Thus $\hat{\Theta}_{w}$ is an algebra homomorphism from $\mathfrak{S} S y m$ onto $\mathcal{W} S y m$.

Remark 5.8 The existence of a surjective algebra homomorphism from SSym not only allows us a shortcut to demonstrating associativity, but leads to an alternate description of the product in the range of that homomorphism. This may be achieved in three steps:

(1) Lifting our $p$ and $q$-tubings in $\mathcal{W}$ Sym to any preimages of the generalized Tonks projection $\Theta_{c}$ on the complete graphs on $p$ and $q$ nodes.

(1) Performing the product of these complete graph tubings in SSym.

(2) Finding the image of the resulting terms under the homomorphism $\hat{\Theta}_{c}$.

This description is independent of our choices of preimages in $\mathfrak{S} S y m$ due to the homomorphism property.

The following corollary follows directly from the properties of the surjective algebra homomorphisms of Theorems 5.6 and 5.7.

Corollary 5.9 WSym is a left $\mathfrak{S}$ Sym-module under the definition $F_{u} \cdot F_{\Theta_{c}(v)}=$ $\hat{\Theta}_{c}\left(F_{u} \cdot F_{v}\right)$ and a right $\mathbf{S}$ Sym-module under the definition $F_{\Theta_{c}(u)} \cdot F_{v}=\hat{\Theta}_{c}\left(F_{u} \cdot F_{v}\right)$. $\mathcal{Y}$ Sym is a left $\mathcal{W}$ Sym-module under the definition $F_{u} \cdot F_{\Theta_{w}}(v)=\hat{\Theta}_{w}\left(F_{u} \cdot F_{v}\right)$ and $a$ right $\mathcal{W}$ Sym-module under the definition $F_{\Theta_{w}(u)} \cdot F_{v}=\hat{\Theta}_{w}\left(F_{u} \cdot F_{v}\right)$. 


\section{Extension to the faces of the polytopes}

Chapoton was the first to point out the fact that in studying the Hopf algebras based on vertices of permutohedra, associahedra and cubes, one need not restrict their attention to just the 0-dimensional faces of the polytopes. He has shown that the Loday-Ronco Hopf algebra Y Sym, the Hopf algebra of permutations SSym, and the Hopf algebra of quasisymmetric functions $\mathcal{Q} S y m$ are each subalgebras of algebras based on the trees, the ordered partitions, and faces of the hypercubes respectively [5]. Furthermore, he has demonstrated that these larger algebras of faces are bi-graded and possess a differential. Here we point out that the cyclohedra-based algebra $\mathcal{W} S y m$ can be extended to a larger algebra based on all the faces of the cyclohedra as well, and conjecture the additional properties.

Chapoton's product structure on the permutohedra faces is given in [5] in terms of ordered partitions. Let $\mathfrak{S} \tilde{S} y m$ be the graded vector space over $\mathbb{Q}$ with the $n$th component of its basis given by the ordered partitions of $[n]$. We write $F_{u}$ for the basis element corresponding to the $m$-partition $u:[n] \rightarrow[m]$, for $0 \leq m \leq n$, and 1 for the basis element of degree 0 . The product in $\mathfrak{S} \tilde{S} y m$ of two basis elements $F_{u}$ and $F_{v}$ for $u:[p] \rightarrow[k]$ and $v:[q] \rightarrow[l]$ is found by summing a term for each shuffle, created by composing the juxtaposition of $u$ and $v$ with the inverse shuffle:

$$
F_{u} \cdot F_{v}=\sum_{\sigma \in S^{(p, q)}} F_{(u \times v) \cdot \sigma^{-1}} .
$$

Here $u \times v$ is the ordered $(k+l)$-partition of $[p+q]$ given by:

$$
(u \times v)(i)= \begin{cases}u(i), & i \in[p], \\ v(i-p)+k, & i \in\{p+1, \ldots, p+q\} .\end{cases}
$$

The bijection between tubings of complete graphs and ordered partitions allows us to write this product geometrically.

Theorem 6.1 The product in $\mathfrak{S} \tilde{S y m}$ may be written as:

$$
F_{u} \cdot F_{v}=\sum_{l \in S^{(p, q)}} F_{\hat{\rho}_{l}(u, v)}
$$

which is just Definition 4.1 extended to all pairs of faces $(u, v)$.

Proof Recall that we found the bijection between tubings of complete graphs and ordered partitions by noting that each tube contains some numbered nodes which are not contained in any other tube. These subsets of $[n]$, one for each tube, make up the partition, and the ordering of the partition is from innermost to outermost tube. Now the Carr-Devadoss isomorphism $\hat{\rho}$ is a bijection of face posets. With this in mind, the same argument applies as in the proof of Theorem 4.1.

In other words, we view our operands (a pair of ordered partitions) as a face of the cartesian product of permutohedra $\mathcal{P}_{p} \times \mathcal{P}_{q}$. Then the product is the formal sum of 

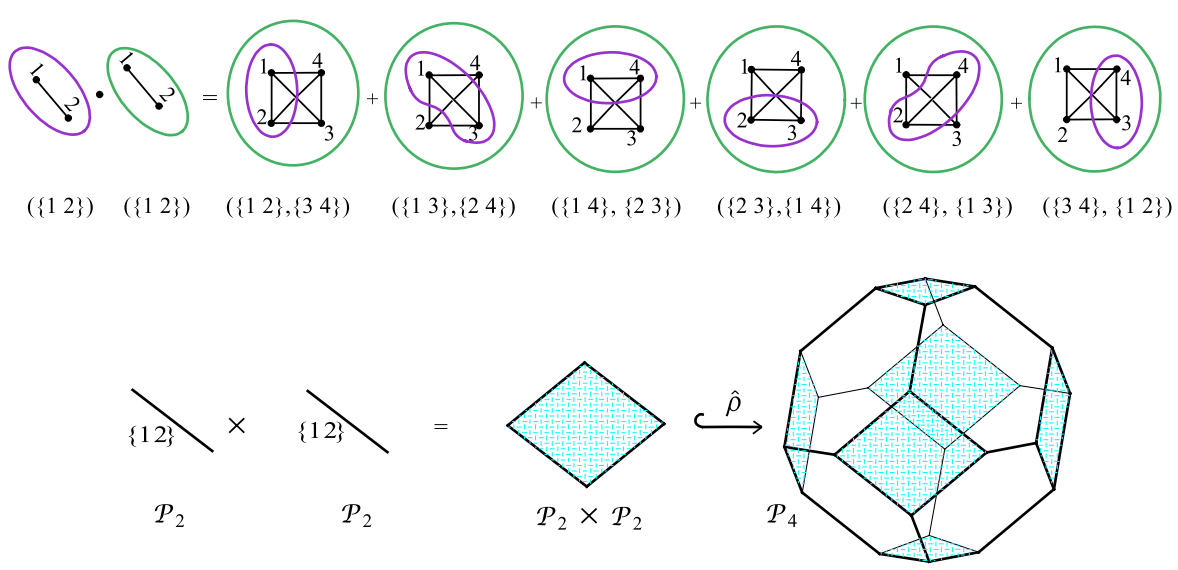

Fig. 20 The product in $\mathfrak{S} \tilde{S y m}$. As an exercise the reader can delete edges in the graphs to illustrate the products in $\mathcal{W} \tilde{S} y m$ and $\mathcal{Y} \tilde{S} y m$

images of that face under the collection of inclusions of $\mathcal{P}_{p} \times \mathcal{P}_{q}$ as a facet of $\mathcal{P}_{p+q}$. An example is in Fig. 20.

We leave to the reader the by now straightforward tasks of finding the geometric interpretations of the coproduct on $\mathfrak{S} \tilde{S} y m$ and of the Hopf algebra structure on the faces of the associahedra, $\mathcal{Y} \tilde{S y m}$. Each one can be done simply by repeating earlier definitions using tubings, but with all sizes of $k$-tubings as operands.

Recall that the faces of the cyclohedra correspond to tubings of the cycle graph. We will define a graded algebra with a basis which corresponds to the faces of the cyclohedra, and whose grading respects the dimensions (plus one) of the cyclohedra.

Definition 6.2 Let $\mathcal{W} \tilde{S} y m$ be the graded vector space over $\mathbb{Q}$ with the $n$th component of its basis given by all the tubings on the cycle graph of $n$ numbered nodes. By $\mathcal{W}_{n}=$ $\mathcal{K} C_{n}$ we denote the poset of tubings on the cycle graph $C_{n}$ with a cyclic numbering of nodes. We write $F_{u}$ for the basis element corresponding to $u \in \mathcal{W}_{n}$ and 1 for the basis element of degree 0 .

The product in $\mathcal{W} \tilde{S} y m$ of two basis elements $F_{u}$ and $F_{v}$ for $u \in \mathcal{W}_{p}$ and $v \in \mathcal{W}_{q}$ is found by summing a term for each shuffle $\iota \in S^{(p, q)}$. First the $l$-tubing $u$ is drawn upon the induced subgraph of the nodes $\iota([p])$ according to the ascending order of the chosen nodes. However, each tube may need to be first broken into several sub-tubes. Then the $m$-tubing $v$ is drawn upon the subgraph induced by the remaining $q$ nodes. However, each of the tubes of $v$ is expanded to also include any of the previously drawn tubes that its nodes are adjacent to.

\section{Definition 6.3}

$$
F_{u} \cdot F_{v}=\sum_{\iota \in S^{(p, q)}} F_{\hat{\rho}_{\iota}\left(\eta_{\iota}\left(\Theta_{\iota}(u)\right), v\right)},
$$

where the facet inclusion $\hat{\rho}_{\iota}$ is the same as in the two previous definitions using this template: Definitions 7.2 and 5.2. 
Theorem 6.4 The product we have just defined makes $\mathcal{W} \tilde{S} y m$ into an associative graded algebra.

Proof The proof is almost precisely the same as for the algebra of vertices of the cyclohedra, $\mathcal{W} S y m$. The only difference is that the tubings do not always have the maximum number of tubes. First we must check that the result of the product is indeed a sum of valid tubings of the cycle graph $C_{p+q}$. We claim that in each term the new tubes we have created are pairwise compatible. The cases to be checked and the reasoning for each are exactly as shown in the proof of Theorem 5.3. Associativity is shown by lifting the tubings to be multiplied to tubings on the complete graphs which are preimages of the extended Tonks projection, and performing the multiplication in Chapoton's algebra. The fact that the extended Tonks projection does preserve the structure here is again an easy check of corresponding terms, following the same pattern as for the $n$-tubings in Theorem 5.6.

Remark 6.5 Chapoton has shown the existence of a differential graded structure on the algebras of faces of the permutohedra, associahedra, and cubes [5]. The basic idea is simple, to define the differential as a signed sum of the bounding sub-faces of a given face. Here we leave for future investigation the possibility of extending this differential to algebras of graph associahedra.

\section{Algebras of simplices}

We introduce a curious new graded algebra whose $n$th component has dimension $n$. We denote it $\Delta S y m$. In fact $\Delta S y m$ may be thought of as a graded algebra whose basis is made up of all the standard bases for Euclidean spaces.

The graph associahedron of the edgeless graph on $n$ vertices is the $(n-1)$-simplex $\Delta^{n-1}$ of dimension $n-1$. Thus the final range of our extension of the Tonks projection is the $(n-1)$-simplex (see Fig. 12). The product of vertices of the permutohedra can be projected to a product of vertices of the simplices. First we define this product by analogy to the previously defined products of tubings of graphs. Then we will show the product to be associative via a homomorphism from the algebra of permutations. Finally we will give a formula for the product using positive integer coefficients and standard Euclidean basis elements.

Definition 7.1 Let $\Delta S y m$ be the graded vector space over $\mathbb{Q}$ with the $n$th component of its basis given by the $n$-tubings on the edgeless graph of $n$ numbered nodes. By $D_{n}$ we denote the set of $n$-tubings on the edgeless graph. We write $F_{u}$ for the basis element corresponding to $u \in D_{n}$ and 1 for the basis element of degree 0 .

\subsection{Graded algebra structure on vertices}

Now we demonstrate a product which respects the grading on $\Delta$ Sym by following the example described above for $\mathfrak{S} S y m$. The product in $\Delta$ Sym of two basis elements $F_{u}$ and $F_{v}$ for $u \in D_{p}$ and $v \in D_{q}$ is found by summing a term for each shuffle $\iota \in S^{(p, q)}$. 


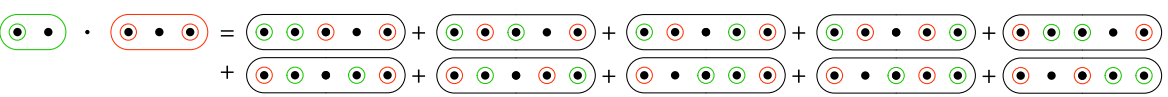

Fig. 21 The product in $\Delta S y m$

Our term of $F_{u} \cdot F_{v}$ will be a $p+q$ tubing of the edgeless graph on $p+q$ nodes. Its tubes will include all the nodes numbered by $\iota([p])$. We denote these by $\overline{\imath(u)}$. In addition we include all the tubes $\hat{\imath}(t)$ for non-universal $t \in v$, and the universal tube. We denote these by $\overline{\hat{\imath}(v)}$. By inspection we see that in this case the union

$$
\overline{\iota(u)} \cup \overline{\hat{\imath}(v)}=\hat{\rho}_{\iota}(\eta(u), v) .
$$

Thus we write:

\section{Definition 7.2}

$$
F_{u} \cdot F_{v}=\sum_{\iota \in S^{(p, q)}} F_{\hat{\rho}_{l}(\eta(u), v)}
$$

Also 1 is a two-sided unit.

An example is shown in Fig. 21.

Theorem 7.3 The product we have just described makes $\Delta$ Sym into an associative graded algebra.

Proof First we must check that the result of the product is indeed a sum of valid $(p+q)$-tubings of the edgeless graph on $p+q$ nodes. This is clearly true, since there will always be only one node that is not a tube in any given term of the product. Associativity is shown by the existence of an algebra homomorphism $5 S y m \rightarrow \Delta S y m$ which we will see next in Lemma 7.5.

Definition 7.4 Let $E_{n}=$ the set of edges of the complete graph on $n$ nodes. Deleting these gives a projection $\Theta_{n}$ from the permutohedra to the simplices, as in Definition 3.4. Then we define $\hat{\Theta}_{\delta}: \mathfrak{S} S y m \rightarrow \Delta$ Sym by

$$
\hat{\Theta}_{\delta}\left(F_{u}\right)=F_{\Theta_{n}}(u)
$$

for $u$ an $n$-tubing of $K_{n}$.

Lemma 7.5 The map $\hat{\Theta}_{\delta}$ is an algebra homomorphism.

Proof The proof follows precisely the same arguments as the proof for Theorem 5.6, except that the cases are simpler. We consider $\hat{\Theta}_{\delta}\left(F_{u} \cdot F_{v}\right)$ for $u \in S_{p}$ and $v \in S_{q}$. We compare the result with $\hat{\Theta}_{\delta}\left(F_{u}\right) \cdot \hat{\Theta}_{E}\left(F_{v}\right)$. Each of the multiplications results in a sum of $\left(\begin{array}{c}p+q \\ p\end{array}\right)$ terms. It turns out that comparing the results of the two operations can 
be done piecewise. Thus we check that for a given shuffle $\iota$ the respective terms of our two operations agree: we claim that

$$
\Theta_{p+q}(\overline{\imath(u)} \cup \overline{\hat{\imath}(v)})=\overline{\iota\left(\Theta_{p}(u)\right)} \cup \overline{\hat{\imath}\left(\Theta_{q}(v)\right)} .
$$

On the left-hand side, $\Theta_{p+q}$ forgets all the information in the tubing it is applied to; except for the following data: which of the nodes is the only node in the universal tube and not in any other tube. This particular node is actually the node numbered by $j=\hat{\imath}\left(v^{-1}(q)\right)$. The effect of $\Theta_{p+q}$ is to create the $n$-tubing of the edgeless graph with node $j$ as the only node that is not a tube.

On the right-hand side $\Theta_{p}$ and $\Theta_{q}$ forget all but the value of $u^{-1}(p)$ and $v^{-1}(q)$ respectively. Then we create the tubing of the edgeless graph by first including all the nodes numbered by $\iota([p])$ and then all the nodes except node $j=\hat{\imath}\left(v^{-1}(q)\right)$.

Remark 7.6 There are clearly algebra homomorphisms $\mathcal{Y}$ Sym $\rightarrow \Delta$ Sym and $\mathcal{W}$ Sym $\rightarrow \Delta$ Sym described by the extended Tonks projections from associahedra and cyclohedra to the simplices.

\subsection{Formula for the product structure}

There is a simple bijection from $n$-tubings of the edgeless graph on $n$ nodes to standard basis elements of $\mathbb{Q}^{n}$. Let $e_{m}^{n}$ be the column vector of $\mathbb{Q}^{n}$ with all zero entries except for a 1 in the $m$ th position. Associate $e_{j}^{n}=e_{u}$ with the $n$-tubing $u$ whose nodes are all tubes except for the $j$ th node. Then use the product of $\Delta S y m$ to define a product of two standard basis vectors of varying dimension: $e_{u} \cdot e_{v}$ is the sum of all $e_{w}$ for $F_{w}$ a term in the product $F_{u} \cdot F_{v}$. Then:

\section{Theorem 7.7}

$$
e_{j}^{p} \cdot e_{l}^{q}=\sum_{i=l}^{p+l}\left(\begin{array}{c}
i-1 \\
l-1
\end{array}\right)\left(\begin{array}{c}
p+q-i \\
q-l
\end{array}\right) e_{i}^{p+q}
$$

Proof Let $e_{l}^{q}=e_{v}$ for the associated tubing $v$. The only node not a tube of $v$ is node $l$. We need only keep track of where $l$ lands under $\hat{\imath}:[q] \rightarrow[p+q]$, where $\hat{\imath}$ is as in Definition 7.2. The only possible images of $l$ are from $l$ to $p+l$, thus the limits of the summation. When $\hat{\imath}(l)=i$ there are several ways this could have occurred. $\hat{\imath}$ must have mapped $[l-1]$ to $[i-1]$ and the set $\{l+1, \ldots, q\}$ to $\{i+1, \ldots, p+q\}$. The ways this can be done are enumerated by the combinations in the sum.

Example 7.8 Consider the example product performed in Fig. 21. Here the formula gives the observed quantities:

$$
e_{2}^{2} \cdot e_{2}^{3}=3 e_{2}^{5}+4 e_{3}^{5}+3 e_{4}^{5} \text {. }
$$




\subsection{Faces of the simplex}

Note that a product of tubings on the edgeless graph (with any number of tubes) is easily defined by direct analogy. Now we present a Hopf algebra with one-sided unit based upon the face posets of simplices. Tubings on the edgeless graphs label all the faces of the simplices. The number of faces of the $n$-simplex, including the null face and the $n$-dimensional face, is $2^{n}$. By adjoining the null face here we thus have a graded bialgebra with $n$th component of dimension $2^{n}$. It would be of interest to compare this with other algebras of similar dimension, such as $\mathcal{Q} S y m$.

Definition 7.9 Let $\Delta \tilde{S y m}$ be the graded vector space over $\mathbb{Q}$ with the $n$th component of its basis given by the tubings on the edgeless graph of $n$ numbered nodes, with one extra basis element included in each component: corresponding to the null facet $\emptyset_{n}$ is the collection consisting of all $n$ of the singleton tubes and the universal tube. By $\mathcal{D}_{n}$ we denote the set of $n$-tubings on the edgeless graph, together with $\emptyset_{n}$. We write $F_{u}$ for the basis element corresponding to $u \in \mathcal{D}_{n}$ and 1 for the basis element of degree 0 .

Now we define the product and coproduct, with careful description of the units.

Let $u \in \mathcal{D}_{p}$ and $v \in \mathcal{D}_{q}$. For a given shuffle $\iota$ our term of $F_{u} \cdot F_{v}$ will be indexed by an element of $\mathcal{D}_{p+q}$ which will include all the nodes numbered by $\iota([p])$. In addition we include all the tubes $\hat{\imath}(t)$ for non-universal $t \in v$, and the universal tube. Thus the product is an extension of Definition 7.2, with a redefined right multiplication by the unit:

\section{Definition 7.10}

$$
F_{u} \cdot F_{v}=\sum_{\iota \in S^{(p, q)}} F_{\hat{\rho}_{l}(\eta(u), v)} ; \quad 1 \cdot F_{u}=F_{u} ; \quad F_{u} \cdot 1=F_{\emptyset_{p}} .
$$

An example is shown in Fig. 22.

The coproduct is defined simply by restricting an element of $\mathcal{D}_{n}$ to its subgraphs induced by the nodes $1, \ldots, i$ and $i+1, \ldots, n$. Given a tubing $u$ of the edgeless graph on $n$ vertices we can find tubings $u_{i}$ and $u_{n-i}$ as follows: for each tube $t \in u$ we find the intersections of $t$ with the two subgraphs (also edgeless) induced by the nodes $1, \ldots, i$ and $i+1, \ldots, n$ respectively.

$$
\Delta\left(F_{u}\right)=\sum_{i=0}^{n} F_{u_{i}} \otimes F_{u_{n-i}} .
$$

An example is shown in Fig. 23.

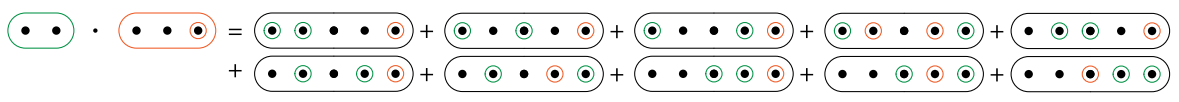

Fig. 22 The product in $\Delta \tilde{S} y m$ 


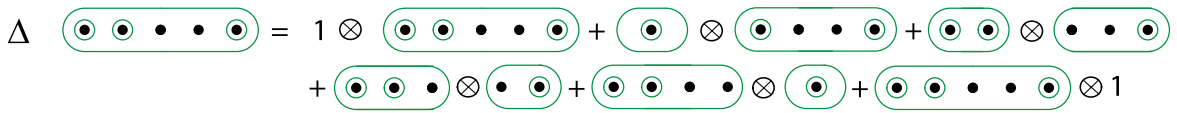

Fig. 23 The coproduct in $\Delta \tilde{S} y m$

Theorem 7.11 The product and coproduct just defined form a graded bialgebra structure on $\Delta \tilde{S y m}$, and therefore a Hopf algebra.

Proof Associativity of the product is due to the observation that the result of multiplying a series of basis elements only depends on the final operand. Coassociativity of the coproduct follows from the fact that terms of both $(1 \otimes \Delta) \Delta F_{u}$ and $(\Delta \otimes 1) \Delta F_{u}$ involve simply splitting the edgeless graph into three consecutive pieces, and restricting $u$ to those pieces. Now we demonstrate that the product and coproduct commute; that is, $\Delta\left(F_{u} \cdot F_{v}\right)=\left(\Delta F_{u}\right) \cdot\left(\Delta F_{v}\right)$. We describe a bijection between the terms on the left-hand and right-hand sides, which turns out to be the identity map. Let $u \in \mathcal{D}_{p}$ and $v \in \mathcal{D}_{q}$. A term of the left-hand side depends first upon a $(p, q)$-shuffle $\iota$ to choose $p$ of the nodes of the $(p+q)$-node edgeless graph. Then after forming the product, a choice of $i \in 0, \ldots, p+q$ determines where to split the result into the pieces of the final tensor product. Let $m=|\{x \in \iota([p]): x \leq i\}|$. Thus $m$ counts the number of nodes in the image of $\iota$ which lie before the split.

To create a term of the right-hand side, we first split both $u$ and $v$, then interchange and multiply the resulting four terms as prescribed in the definition of the tensor product of algebras. Our matching term on the right-hand side is the one formed by first splitting $u$ after node $m$, and $v$ after node $i-m$. Then in the first multiplication choose the $(m, i-m)$-shuffle $\sigma(x)=\iota(x)$, and in the second use the $(q-m, q-$ $i+m)$-shuffle $\sigma^{\prime}(x)=\iota(x+m)-i$. These choices define an identity map from the set of terms of the left-hand side to those on the right.

Remark 7.12 $\Delta \tilde{S y m}$ is closely related to the free associative trialgebra on one variable described by Loday and Ronco in [12]. In [12, Proposition 1.9] the authors describe the products for that trialgebra. Axiomatically, the first two products automatically form a dialgebra. The sum of these two products appears as two of the terms of our shuffle-based product! We leave it to an interested reader to uncover the precise relationship, perhaps duality, between the two structures.

Acknowledgements We would like to thank the referees for helping us make connections to other work, and for making some excellent suggestions about the presentation of the main ideas. We also thank the following for tutorials and helpful conversations on the subject matter: Satyan Devadoss, Aaron Lauve, Maria Ronco and Frank Sottile.

\section{References}

1. Aguiar, M., Sottile, F.: Structure of the Malvenuto-Reutenauer Hopf algebra of permutations. Adv. Math. 191(2), 225-275 (2005). MR2103213 (2005m:05226)

2. Aguiar, M., Sottile, F.: Structure of the Loday-Ronco Hopf algebra of trees. J. Algebra 295(2), 473511 (2006). MR2194965 (2006k:16078) 
3. Bott, R., Taubes, C.: On the self-linking of knots. J. Math. Phys. 35(10), 5247-5287 (1994). Topology and physics. MR1295465 (95g:57008)

4. Carr, M.P., Devadoss, S.L.: Coxeter complexes and graph-associahedra. Topol. Appl. 153(1-2), 21552168 (2006). MR2239078 (2007c:52012)

5. Chapoton, F.: Bigèbres différentielles graduées associées aux permutoèdres, associaèdres et hypercubes. Ann. Inst. Fourier (Grenoble) 50(4), 1127-1153 (2000). MR1799740 (2002f:16081)

6. Devadoss, S., Forcey, S.: Marked tubes and the graph multiplihedron. Algebraic Geom. Topol. 8(4), 2081-2108 (2008). MR2460880

7. Devadoss, S.L.: A space of cyclohedra. Discrete Comput. Geom. 29(1), 61-75 (2003). MR1946794 (2003j:57027)

8. Devadoss, S.L.: A realization of graph associahedra. Discrete Math. 309(1), 271-276 (2009). MR2479448

9. Forcey, S., Lauve, A., Sottile, F.: Constructing cofree compositional coalgebras (manuscript in preparation)

10. Hohlweg, C., Lange, C.E.M.C.: Realizations of the associahedron and cyclohedron. Discrete Comput. Geom. 37(4), 517-543 (2007). MR2321739 (2008g:52021)

11. Loday, J.-L., Ronco, M.O.: Hopf algebra of the planar binary trees. Adv. Math. 139(2), 293-309 (1998). MR1654173 (99m:16063)

12. Loday, J.-L., Ronco, M.O.: Trialgebras and families of polytopes. In: Homotopy Theory: Relations with Algebraic Geometry, Group Cohomology and Algebraic $K$-Theory. Contemp. Math., vol. 346, pp. 369-398. Am. Math. Soc., Providence (2004). MR2066507 (2006e:18016)

13. Malvenuto, C., Reutenauer, C.: Duality between quasi-symmetric functions and the Solomon descent algebra. J. Algebra 177(3), 967-982 (1995). MR1358493 (97d:05277)

14. Markl, M.: Simplex, associahedron, and cyclohedron. In: Higher Homotopy Structures in Topology and Mathematical Physics, Poughkeepsie, NY, 1996. Contemp. Math., vol. 227, pp. 235-265. Am. Math. Soc., Providence (1999). MR1665469 (99m:57020)

15. Morton, J., Shiu, A., Pachter, L., Sturmfels, B.: The cyclohedron test for finding periodic genes in time course expression studies. Stat. Appl. Genet. Mol. Biol. 6 (2007), Art. 21, 25 pp. (electronic). MR2349914

16. Postnikov, A., Reiner, V., Williams, L.: Faces of generalized permutohedra. Doc. Math. 13, 207-273 (2008). MR2520477

17. Postnikov, A.: Permutohedra, associahedra, and beyond. Int. Math. Res. Not. IMRN 6, 1026-1106 (2009). MR2487491

18. Reading, N.: Cambrian lattices. Adv. Math. 205(2), 313-353 (2006). MR2258260 (2007g:05195)

19. Simion, R.: A type-B associahedron. Adv. Appl. Math. 30(1-2), $2-25$ (2003). Formal power series and algebraic combinatorics (Scottsdale, AZ, 2001). MR1979780 (2004h:52013)

20. Tonks, A.: Relating the associahedron and the permutohedron. In: Operads: Proceedings of Renaissance Conferences, Hartford, CT/Luminy, 1995. Contemp. Math., vol. 202, pp. 33-36. Am. Math. Soc., Providence (1997). MR1436915 (98c:52015) 